OPEN ACCESS

Edited by:

Linhua Zhao,

China Academy of Chinese Medical

Sciences, China

Reviewed by:

Joseph Selvin,

Pondicherry University, India

Chenyang Lu,

Ningbo University, China

*Correspondence:

Jianguo Tang

tangjianguo@5thhospital.com

Specialty section:

This article was submitted to Microbiome in Health and Disease,

a section of the journal

Frontiers in Cellular and

Infection Microbiology

Received: 18 July 2021 Accepted: 13 December 2021 Published: 07 January 2022

Citation:

Hu W, Huang L, Zhou Z, Yin L and Tang J (2022) Diallyl Disulfide (DADS)

Ameliorates Intestinal Candida albicans Infection by Modulating the Gut Microbiota and Metabolites and Providing Intestinal Protection in Mice. Front. Cell. Infect. Microbiol. 11:743454.

doi: 10.3389/fcimb.2021.743454

\section{Diallyl Disulfide (DADS) Ameliorates Intestinal Candida albicans Infection by Modulating the Gut microbiota and Metabolites and Providing Intestinal Protection in Mice}

\author{
Wanchao Hu, Liou Huang, Ziyang Zhou, Liping Yin and Jianguo Tang* \\ Department of Trauma-Emergency \& Critical Care Medicine, Shanghai Fifth People's Hospital, Fudan University, \\ Shanghai, China
}

Diallyl disulfide (DADS), a garlic extract also known as allicin, has been reported to have numerous biological activities, including anticancer, antifungal, and inflammation-inhibiting activities, among others. Although many studies have assessed whether DADS can treat Candida albicans infection in vitro, its in vivo function and the underlying mechanism are still not clear. Accumulated evidence has implicated the gut microbiota as an important factor in the colonization and invasion of $C$. albicans. Thus, this study aimed to identify the mechanism by which DADS ameliorates dextran sulfate (DSS)-induced intestinal C. albicans infection based on the systematic analysis of the gut microbiota and metabolomics in mice. Here, we determined the body weight, survival, colon length, histological score, and inflammatory cytokine levels in the serum and intestines of experimental mice. Fecal samples were collected for gut microbiota and metabolite analysis by $16 \mathrm{~S}$ rRNA gene sequencing and LC-MS metabolomics, respectively. DADS significantly alleviated DSS-induced intestinal C. albicans infection and altered the gut microbial community structure and metabolic profile in the mice. The abundances of some pathogenic bacteria, such as Proteobacteria, Escherichia-Shigella, and Streptococcus, were notably decreased after treatment with DADS. In contrast, SCFA-producing bacteria, namely, Ruminiclostridium, Oscillibacter, and Ruminococcaceae_UCG-013, greatly increased in number. The perturbance of metabolites in infectious mice was improved by DADS, with increases in secondary bile acids, arachidonic acid, indoles and their derivatives, which were highly related to the multiple differentially altered metabolic pathways, namely, bile secretion, arachidonic acid metabolism, and tryptophan metabolism. This study indicated that DADS could modulate gut microbiota and metabolites and protect the gut barrier to alleviate DSS-induced intestinal C. albicans infection in mice. Moreover, this work might also provide novel insight into the treatment of C. albicans infection using DADS.

Keywords: diallyl disulfide, Candida albicans, gut barrier, gut microbiota, gut metabolites 


\section{INTRODUCTION}

Candida albicans is one of the most common commensal fungi in humans but causes millions of disseminated infections each year and can even cause death (Wisplinghoff et al., 2004; Bongomin et al., 2017; Pappas et al., 2018; Toda et al., 2019). As an opportunistic pathogen, C. albicans is a normal component of the human gut microbiota. Several studies have shown that the gut community could influence the colonization and invasion of C. albicans (Mason et al., 2012; Gutierrez et al., 2020). Moreover, gut C. albicans can also induce bacteremia and an imbalance in the gut microbiota (Neville et al., 2015; Hiengrach et al., 2019; Valentine et al., 2019). Therefore, symptomatic C. albicans infection is closely related to the interplay of C. albicans with other gut bacteria. On the other hand, damage to the intestinal barrier also plays an important role in gut microbiota dysbiosis and fungal disease (Yan et al., 2013; Basmaciyan et al., 2019; Kumamoto et al., 2020). Therefore, it is of great importance to understand the factors affecting fungal colonization in order to prevent disease associated with $C$. albicans infection. However, when we establish intestinal $C$. albicans infectious models, mice will excrete gavaged C. albicans and cannot be infected. Thus, we used DSS to induce the gut damage and then gavage C. albicans (Hiengrach et al., 2019). In our previous studies, we found that cocultivation of pathogenic Escherichia coli with C. albicans could reduce the colonization of intestinal cells by $C$. albicans and reduce fungal virulence gene expression (Yang et al., 2016). An increasing number of studies have indicated that the intestinal microbiota, metabolites, and gut barrier could affect the colonization and invasion of $C$. albicans. Previous studies using 16S rRNA sequencing method suggested that intestine-derived C. albicans can erode the intestinal mucosa by regulating the intestinal flora. Moreover, compared with that in healthy mice, the relative abundances of Bacteroides, Pseudomonas, and Enterococcus were increased significantly, while the abundance of Firmicutes (such as Lactobacillus) was decreased, in model mice with intestinal $C$. albicans infection. In addition, C. albicans has a synergistic pathogenic effect with Enterococcus, which can lead to the destruction of the epithelial barrier by reducing the expression of the intestinal epithelial adhesion protein E-cadherin. Additionally, intestinal injury is a prerequisite for disseminated gut C. albicans infection (Koh et al., 2008; Bertolini et al., 2019; Hiengrach et al., 2019; Zhai et al., 2020). In addition, the gut microbiota and metabolites are closely related. Nontargeted high-throughput metabolomics analysis technologies provide an opportunity to explore the changes in metabolites related to microbiota community imbalance during disease development. As a part of the gut microbiome, $C$. albicans could also produce some toxic metabolites, such as adhesin and extracellular proteases (Staniszewska, 2020). Moreover, the impact of C. albicans on gut flora will also cause changes in the microbiota metabolites, but there are not many studies in this area. Studies indicated that fungi, as a kind of commensal fungi, could promote the production of indole derivatives, such as, tryptophan and indole-3-aldehyde, which could activate AhR to protect and maintain mucosal integrity during fungal infections or chemical damage and induce anticandidal resistance (Heath-Pagliuso et al., 1998; Wikoff et al., 2009; Zelante et al., 2013; Bessede et al., 2014; Romani et al., 2015). Studies have shown that inflammatory bowel disease (IBD) is related to changes in microorganisms and the metabolic environment in the colon, which participate in signal transduction and immune system regulation and affect the activity of antibiotics (Machiels et al., 2014; Imhann et al., 2018; Franzosa et al., 2019). Some metabolites produced by gut bacteria, such as short-chain fatty acids (SCFAs), indoles, bile acids, and amino acids, can regulate the intestinal epithelium and immune function (Sun et al., 2017; Parada Venegas et al., 2019; Lavelle and Sokol, 2020). However, few researchers have combined studies of the intestinal microbiota with studies of the changes in fecal metabolites during intestinal C. albicans infection.

Other interesting studies have indicated that traditional Chinese medicines have a certain effect in regulating gut microbiota. Moreover, diet plays an important role in human health through regulating gut microbiome (Gentile and Weir, 2018; Zmora et al., 2019). Garlic, as a food that is often consumed daily, is also a longstanding commonly used Chinese folk medicine. The effective ingredient in garlic is diallyl disulfide (DADS), also known as allicin, which can exert antifungal, antibacterial, and antitumor effects and ameliorate cardiovascular disease (Yi and Su, 2013; He et al., 2021). The antifungal effect of DADS against C. albicans involves the inhibition of biofilm formation by preventing the conversion of yeast to hyphae (Khodavandi et al., 2011). Additionally, DADS can reduce oxidative stress and inflammation and inhibit the cell apoptosis induced by C. albicans (Lemar et al., 2005). Moreover, DADS also has important effects on cellular immunity and humoral immunity (Yadegari et al., 2009). However, animal experiments involving the treatment of gut $C$. albicans infection with DADS have not yet been reported, and whether the treatment mechanism involves the gut microbiota and the metabolite intestinal barrier has not been studied. In this study, the mechanism of DADS treatment was explored by establishing mouse models with DSS-induced intestinal C. albicans infection, which are more representative of the conditions in the human body (Hiengrach et al., 2019).

\section{MATERIALS AND METHODS}

\section{Animals and C. albicans Culture}

Female C57BL/6 mice aged 8 weeks were purchased and housed in the Animal Center of East China Normal University (Shanghai, China). Mice were fed ad libitum and allowed to adapt to the environment $\left(24 \pm 2{ }^{\circ} \mathrm{C}, 60 \pm 5 \%\right.$ relative humidity, $12 / 12 \mathrm{~h}$ dark/light cycle) for one week. All animal experiments were approved by the Experimental Animal Ethical Review Committee, East China Normal University (Shanghai, China). C. albicans (strain SC5314) was purchased from the China General Microbiological Culture Collection Center (CGMCC) and then cultivated in yeast extract peptone dextrose (YEPD) liquid medium. Next, a loop was inoculated and streaked on chromogenic medium for the detection of $C$. albicans (CHROMagar Company, France). Then, a single colony was streaked on a YEPD agar plate, incubated for $25 \mathrm{~h}$ at $35^{\circ} \mathrm{C}$ and reidentified by mass spectrometry (Shanghai Fifth People's Hospital, Fudan University, Department of Laboratory). An 
inoculum of $1.0 \times 10^{6} \mathrm{C}$. albicans cells was prepared in $0.3 \mathrm{ml}$ phosphate buffered saline (PBS, $\mathrm{pH} 7.4$ ).

\section{Induction of Intestinal C. albicans Infection in a Mouse Model With DSS}

Mouse models of intestinal $C$. albicans infection were constructed as previously published (Hiengrach et al., 2019). Dextran sulfate (DSS; 3\% wt/vol, $40 \mathrm{kD}$, Sigma-Aldrich, USA) was included in the drinking water throughout the entire process to induce colon damage. The PBS yeast suspension was orally administered every 3 days to promote $C$. albicans colonization in the gut (Figure 1A). Repeated oral-gastric gavage of C. albicans was performed to maintain the fungal load in the gut at a certain level. The fecal $C$. albicans content was evaluated on chromogenic medium (Figure 1B). To determine the optimum treatment effect, we tested different concentrations of DADS to treat C. albicans infectious mice; 6,20 , and $40 \mathrm{mg} / \mathrm{kg}$ were selected based on previous publications (Benavides et al., 2007; Yousuf et al., 2011; Alam et al., 2013; Liang et al., 2015; Motta et al., 2015; Zhang et al., 2019). DADS was diluted in $0.3 \mathrm{ml}$ PBS and administered by oral-gastric gavage after $6 \mathrm{~h}$ of treatment with C. albicans (Figure 1C). A dosage of $20 \mathrm{mg} / \mathrm{kg}$ DADS was determined to be suitable for subsequent experimental groups through an evaluation of survival rate, colon length and disease activity index (Figure 2). Seventy-five female mice were randomly divided into five groups: control, DSS, CA + DSS, $\mathrm{CA}+\mathrm{DSS}+\mathrm{DADS}(20 \mathrm{mg} / \mathrm{kg})$, and DSS + DADS. After fifteen days of treatment, feces were collected from all mice and stored at $-80^{\circ} \mathrm{C}$. Blood was sampled by retro-orbital puncture, after which the mice were sacrificed by cervical dislocation and their colons and spleens were harvested.

\section{Histomorphological Analysis}

Colon samples were fixed in $4 \%$ neutral paraformaldehyde solution, dehydrated, embedded in paraffin, sectioned into 5$\mu \mathrm{m}$-thick slices and then stained with hematoxylin and eosin (H\&E) for observation. Histological changes were assessed by two blinded experienced pathologists at the same time using a previously described scoring system (Williams et al., 2001; Christophi et al., 2016), and the average score was taken.

\section{Western Blot Analysis}

To analyze the protein expression of colon tissues, western blot analysis was performed according to standard methods. Primary antibodies against Occludin (Proteintech, 13409-1-AP), Claudin-1 (GeneTex, GTX54539), and $\beta$-actin (Cell Signaling

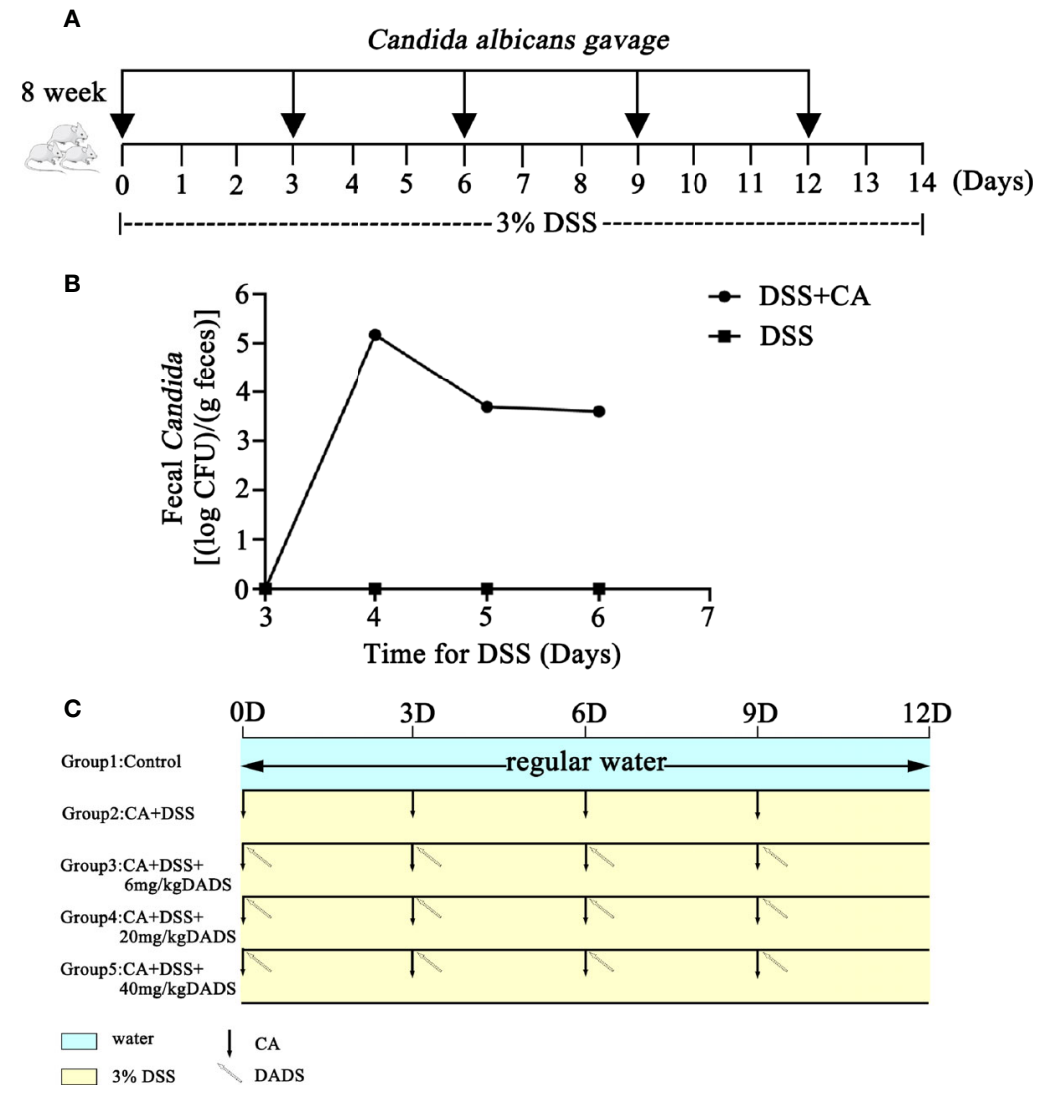

FIGURE 1 | Schematic of the animal experiment design, including C. albicans gavage and DADS administration. (A) Schematic of C. albicans infection in the animal models. (B) Fungal burden in feces. (C) Schematic diagram of the experiment to compare the function of different concentrations of DADS. 
A

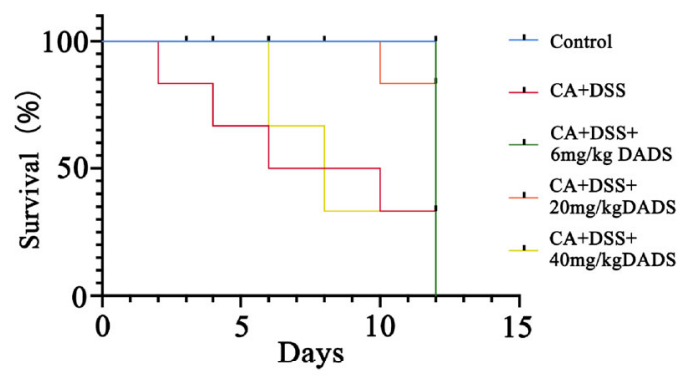

B

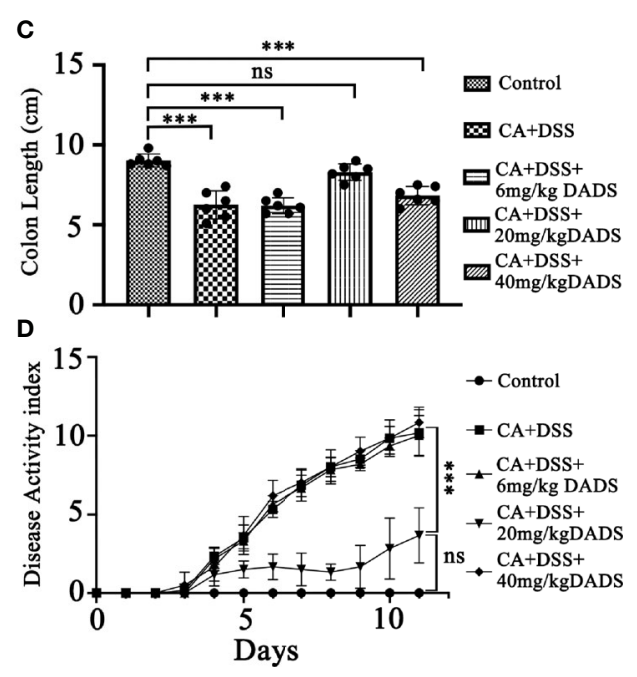

FIGURE 2 | Effects of different concentrations of DADS on mice with C. albicans infection. (A) Survival study. (B, C) Colon length and relevant statistical analysis. (D) The disease activity index of animals in each group. Data are presented as the mean $\pm \operatorname{SEM}(n=6) .{ }^{\star} P<0.05,{ }^{* \star} P<0.01,{ }^{\star \star \star} P<0.001$.

Technology, CST-3077) were used. The secondary antibodies were obtained from Jackson ImmunoResearch company: antimouse IgG (115-035-003) and anti-rabbit IgG (111-035-003).

Then, the protein bands were visualized using an ECL chemiluminescence imaging system and quantified by ImageJ software to calculate the ratios of IntDen (target protein)/IntDen $(\beta$-actin).

\section{Enzyme-Linked Immunosorbent Assays}

Mouse blood samples were collected and then centrifuged at 3,000 $\mathrm{rpm}$ at $4^{\circ} \mathrm{C}$ for $10 \mathrm{~min}$ for serum collection. Colon tissues were ground in $9 \times$ fold homogenization medium and then centrifuged for $10 \mathrm{~min}$ at $3,000 \mathrm{rpm}$ and $4^{\circ} \mathrm{C}$ to collect the supernatants. All serum and tissue supernatants were stored at $-80^{\circ} \mathrm{C}$ for later simultaneous detection. Subsequently, the levels of IL- 6 and IFN- $\gamma$ were measured by murine ELISA kits (88-7064, Thermo Fisher, Austria; EK280/3-01, MuLTI SCIENCE, Shanghai) according to the manufacturer's instructions.

\section{Measurement of FITC-Dextran Leakage}

As previously described (Watson et al., 2015), FITC-dextran leakage was measured to evaluate gut permeability. Briefly, mice were starved overnight for approximately $8 \mathrm{~h}$ and then administered $25 \mathrm{mg} / \mathrm{ml}$ FITC-dextran (4 kDa, Sigma-Aldrich,
USA) dissolved in PBS. FITC-dextran was gavaged at a dose of $0.6 \mathrm{mg}$ per gram of body weight. Blood samples $(400 \mu \mathrm{l})$ were collected via retro-orbital puncture after $4 \mathrm{~h}$. Then, the supernatants were collected by centrifugation and mixed with an equivalent amount of PBS. Afterward, the fluorescence intensity of diluted serum $(100 \mu \mathrm{l})$ from each sample was detected using a multimode reader (excitation: $485 \mathrm{~nm}$ emission: $528 \mathrm{~nm}$, bandwidth: $20 \mathrm{~nm}$ ). The quantity of FITC was calculated with a standard curve.

\section{S rRNA Analysis of the Microbial Community}

The composition of the gut microbiota and profile of metabolites were assessed as previously described (Hu et al., 2021). Feces were collected from each group of mice. Then, we randomly selected 6 fecal pellets for microbiome and metabolomics analyses. Briefly, DNA was extracted from feces by a Standard DNA Extraction Kit (QIAGEN). Then, the quality and quantity of DNA were confirmed by agarose gel electrophoresis. The V3-V4 regions of the 16S rRNA genes were amplified, and the quality was verified; the amplicon was then purified and amplified again. Sequencing of the V3-V4 gene amplicons was obtained using the Illumina MiSeq platform. The raw data were filtered, and clean tags were removed to obtain valid tags for preparing operational taxonomic 
units (OTUs), which were classified using Vsearch software (version 2.4.2) (Rognes et al., 2016) with a threshold of $97 \%$ sequence similarity. Subsequently, according to the sequence comparison of OTUs, pynast (v0.1) software (Caporaso et al., 2010) was used to construct a phylogeny. The diversity and composition of the intestinal microbiota were determined based on a rarefied OTU table. Alpha diversity indexes of fecal samples were generated from a normalized OTU table at a uniform depth. Beta diversity indexes were generated to determine whether significant differences in gut microbiota existed among different groups based on the Bray-Curtis algorithm and unweighted UniFrac distance and were also determined by principal component analysis (PCA).

\section{Fecal Metabolome Analysis}

LC-MS analysis was performed by OE BioTech (Shanghai, China). Fecal sample preparation and analysis were performed as previously described (Liu et al., 2020). Briefly, fecal pellets (60 mg) were mixed with $500 \mu \mathrm{l}$ of solvent and then ground, vortexed and centrifuged for $15 \mathrm{~min}$ at $13,000 \mathrm{rpm}$ at $4^{\circ} \mathrm{C}$. Subsequently, the supernatant was filtered using a $0.22 \mu \mathrm{m}$ microfilter, and the resulting supernatant was stored at $-80^{\circ} \mathrm{C}$ for LC-MS analysis. The quality control (QC) group was established by pooling equal volumes of supernatant from each sample to determine whether the mass spectrum platform of the system was stable during the whole experiment. The metabolite profiles were analyzed on an AB TripleTOF 6600 mass spectrometer (AB Sciex) combining ESI sources in both positive and negative ion scan modes. TOF parameters were as described previously (Xiong et al., 2019). All reagents used were of high-performance liquid chromatography (HPLC) grade.

The LC-MS data from fecal pellets were processed by Progenesis QI software (Waters Corporation, Milford, USA), and then Progenesis QI Data Processing Software was used to identify the metabolites. The normalized data were visualized by PCA and orthogonal partial least squares-discriminant (OPLSDA) analysis using the ropls package in R. The ellipses in PCA and OPLS-DA plots were employed to characterize metabolic perturbation among groups in a Hotelling T2 region with a 95\% confidence interval threshold.

The variable importance in projection (VIP) was calculated based on the OPLS-DA model to identify significant metabolites with a VIP $>1.0$ and $P$-value $<0.05$. The KEGG (http://www.kegg. $\mathrm{com} /$ ) database was used to explore the related metabolic pathways.

\section{Statistical Analysis}

Statistical analysis was carried out by SPSS 24.0 software (SPSS Inc., Chicago, IL). All data were calculated from no fewer than three replicates and are presented as the mean \pm S.E.M. All data were tested for normal distribution before comparisons between groups. If the data were normally distributed, the differences between groups were examined for statistical significance by Student's t-test (for comparisons between two groups) or oneway ANOVA (for comparisons between multiple groups). Otherwise, nonparametric tests, including the Wilcoxon test, Bray-Curtis distance, Euclidean distance, and UniFrac, were used to analyze the gut microbiota and metabolite data.
Survival analysis was performed by log-rank test. The analysis methods used are provided in the figure legends. A $P$-value $<0.05$ was considered statistically significant.

\section{RESULTS}

\section{DSS-Induced Intestinal C. albicans Infection Alleviated by $20 \mathrm{mg} / \mathrm{kg}$ DADS in Mice}

We evaluated whether DADS could exert antifungal and antiinflammatory effects in gut C. albicans infectious mouse models (Figure 1). The fecal C. albicans level was maintained at $10^{4}$ $\mathrm{CFU} / \mathrm{g}$ feces after treatment with $3 \%$ DSS and repeated gavage with C. albicans (Figures 1A, B).

Infected mice were treated with three concentrations of DADS: 6, 20, and $40 \mathrm{mg} / \mathrm{kg}$ (Figure 1C). Mice with intestinal C. albicans infection exhibited a decreased survival rate and shortened colon, both of which were improved by administration of 6 and $20 \mathrm{mg} / \mathrm{kg}$ DADS but not $40 \mathrm{mg} / \mathrm{kg}$ DADS (Figures 2A-C). Meanwhile, treatment with $20 \mathrm{mg} / \mathrm{kg}$ DADS caused a reduction in the disease activity index (DAI), consistent with the findings of weight loss, general condition and fecal occult blood tests (Figure 2D). From the observations above, $20 \mathrm{mg} / \mathrm{kg}$ was regarded as the optimum concentration of DADS for the following experimental grouping (Figure 3A). Mice treated with DSS to induce colon damage were considered the model control group (DSS), and mice treated with DSS + DADS were the experimental group. While administration DSS and C. albicans significantly decreased the body weight and survival of mice, DADS treatment alleviated these effects (Figures 3B, C). Moreover, DADS treatment caused a marked decrease in the load of fecal C. albicans compared with the CA + DSS group (Figures 3D, E). These data suggested that it is better to alleviate DSS-induced intestinal C. albicans infection in mice with $20 \mathrm{mg} / \mathrm{kg}$ DADS treatment, compared with $6 \mathrm{mg} / \mathrm{kg}$. As for $40 \mathrm{mg} /$ $\mathrm{kg}$ DADS, there is no improvement.

\section{DADS Exhibited an Anti-Inflammatory Effect and Protected the Epithelial Barrier of Mice With DSS-Induced Intestinal C. albicans Infection}

Spleen size can reflect inflammation in mice. DSS and C. albicans increased the spleen/body weight ratio, which was significantly improved by DADS (Figures 4A, B). In addition, we employed ELISA to quantify the expression of proinflammatory cytokines, namely, IL-6 and IFN- $\gamma$. Notably, DSS and C. albicans caused a significant increase in IL- 6 and IFN- $\gamma$ in both the serum and colon, and these changes could be reversed by DADS treatment (Figures 4C, D). To investigate the effect of DADS on intestinal inflammation, the length of colons and histological changes were compared among groups. Consistent with the above results, DADS ameliorated the colon shortening observed in mice treated with DSS and C. albicans (Figures 5A, B). The histological examination results revealed partial loss of the mucosal glands, erosion of the submucosal and muscular layers, and increased neutrophil infiltration in mice with C. albicans infection, which was 

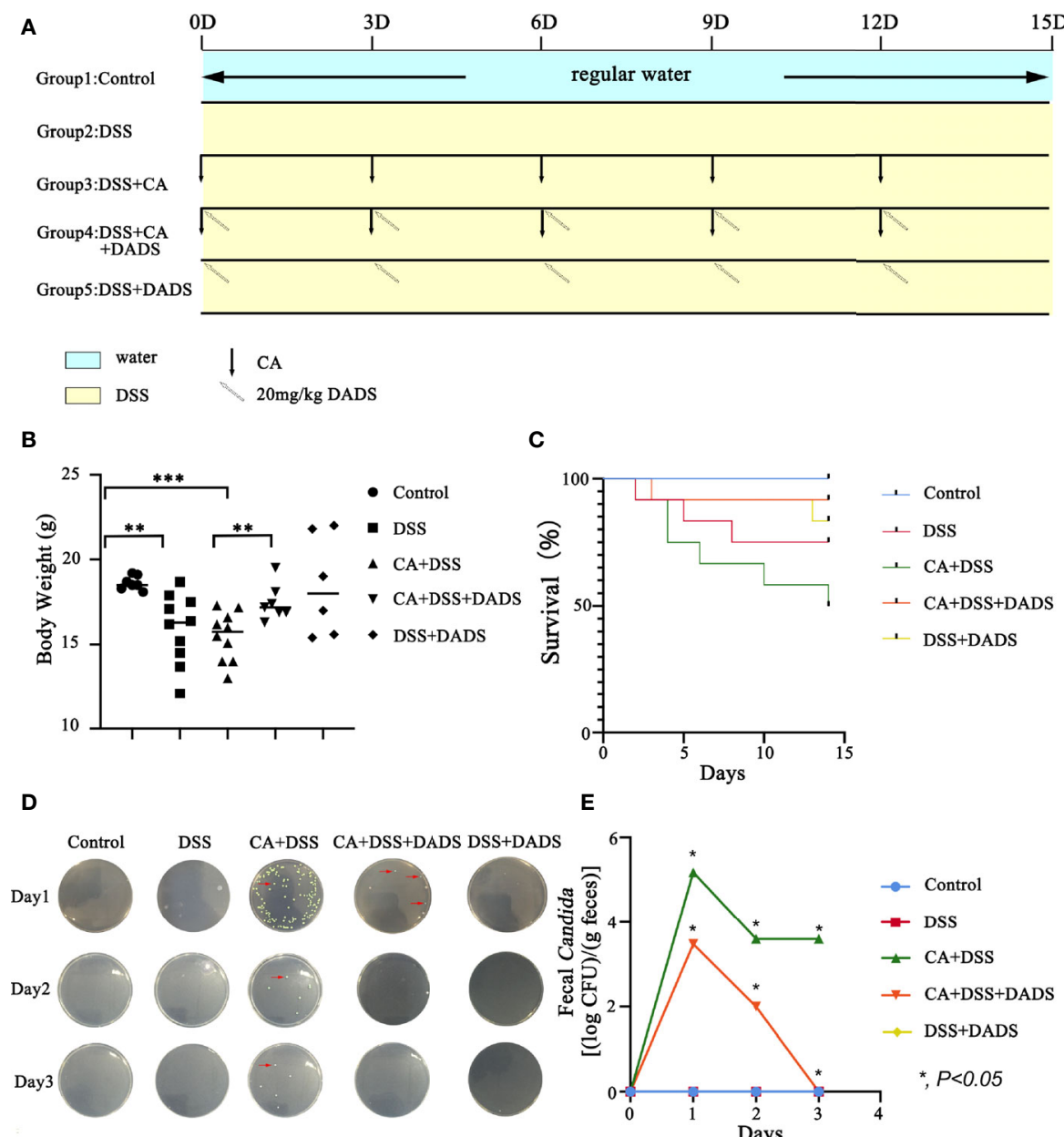

E

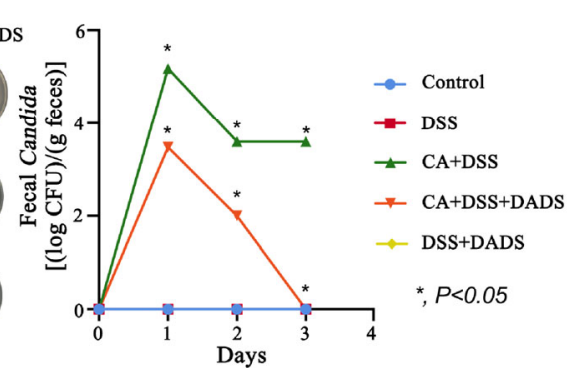

FIGURE 3 | DADS alleviated symptoms in mice with C. albicans infection. (A) Schematic of C. albicans gavage infection and $20 \mathrm{mg} / \mathrm{kg}$ DADS administration. (B) Changes in body weight. (C) Survival study. (D, E) Fungal burden in feces.

alleviated by DADS (Figures 5C, D). Damage to the intestine was also evaluated by quantifying the FITC content in the serum, which indicated that the increased permeability in the DSS and CA + DADS groups could be improved by DADS (Figure 5E). Furthermore, the expression levels of the tight junction proteins Claudin-1 and Occludin in the colon were also quantitatively analyzed; these proteins were significantly depleted in intestinal $C$. albicans-infected mice and improved in the CA + DSS + DADS group (Figures 5F, G). These data demonstrated that DADS treatment could ameliorate the damage to the intestinal barrier caused by DSS and C. albicans.

\section{DADS Altered the Gut Microbiota Community Composition of Mice With DSS-Induced Intestinal C. albicans Infection}

Many studies have indicated that certain gut microbiota could influence the survival and colonization properties of C. albicans. During inflammation of the colon and DADS treatment, the gut microbial communities may be altered. Based on this model, 16S rRNA gene sequencing was used to identify key bacteria and relevant metabolic pathways that might be changed among groups. More than $99.4 \%$ of the sequence exhibited good coverage values, which indicated adequate sequencing depth for all groups. All other alpha diversity values (OTUs, observed species, Chao1, and Shannon index) were lower in mice after treatment with DSS and/or C. albicans than in the control group and were not improved by DADS (Table 1). PCA showed that $C$. albicans, DSS, and DADS treatment induced changes in the intestinal microbiota composition. Moreover, the fecal samples from the DSS and CA + DSS groups were clustered together with high similarity, but in DADS-treated mice, samples from the $\mathrm{CA}+\mathrm{DSS}+\mathrm{DADS}$ group were clustered with the control samples (Figure 6A). PCA also separated the CA + DSS and CA + DSS + DADS groups, which indicated that these gut microbial communities were significantly different (Figure 6B). As shown in Figures 3C, D, we determined the relative abundances of the top 15 bacteria at the genus level among the groups. The genera 

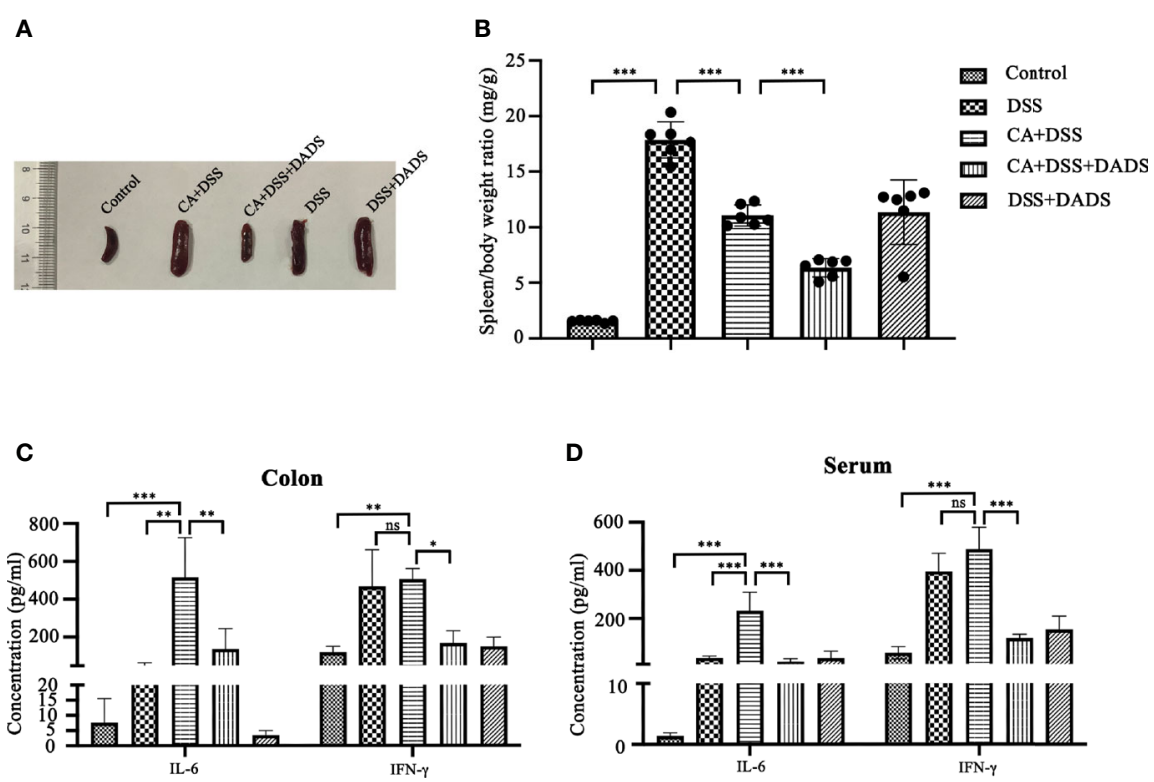

FIGURE 4 | DADS exhibited an anti-inflammatory effect in mice with C. albicans infection. (A, B) Spleen size and the spleen/body weight ratio. (C, D) The concentrations of IL-6 and IFN- $\gamma$ in the colon and serum.

Bacteroides, Escherichia-Shigella, Lachnospiraceae_ NK4A136_group, Parabacteroides, and others exhibited high relative abundance in all groups. Compared with $C$. albicansinfected mice, the DADS treatment group had decreased relative abundances of Escherichia-Shigella and Parabacteroides.

The key bacteria were visualized by a heatmap and analyzed by the Wilcoxon rank-sum test (Figures 7A, B). At the phylum level, DADS treatment was associated with a decreased abundance of Proteobacteria $(P=0.019$, Figure $7 C)$ and increase abundance of Tenericutes $(P=0.002$, Figure $7 D)$ compared to the intestinal C. albicans infectious group $(\mathrm{P}<0.05)$ (Figure 7A). As shown in Figure 7B, at the genus level, the abundances of EscherichiaShigella $(P=0.014$, Figure $7 \mathbf{E})$, Faecalibacterium $(P=0.027$, Figure 7F), Parabacteroides $(P=0.001$, Figure 7G), and Streptococcus $(P=0.023$, Figure $7 \mathbf{H})$ were significantly decreased, and the abundances of Prevotellaceae_NK3B31_group $(P=0.002$, Figure 7I), Ruminiclostridium $(P=0.023$, Figure $7 \mathbf{J})$, Ruminococcaceae_UCG-013 ( $P=0.002$, Figure 7K), and Oscillibacter $(P=0.038$, Figure $7 \mathbf{L})$ were enriched in DADStreated mice (Table 2).

\section{DADS Improved the Fecal Metabolite Profiles of Mice With DSS-Induced Intestinal C. albicans Infection}

Metabolic changes are closely related to alterations of the gut microbiota and are also considered a crucial hallmark of intestinal inflammation (Lanis et al., 2017). Thus, we performed LC-MS analysis to detect differentially expressed metabolites and relevant key metabolic pathways among groups. A total of 8,689 metabolites were identified in 30 fecal samples among the five groups. The PCA scatter plots showed clustered QC samples, which indicated the high quality of metabolomics analysis (Figure 8A). We further identified the differences in the metabolic profile between the CA + DSS and 20 $\mathrm{mg} / \mathrm{kg}$ DADS treatment groups using two-dimensional PCA, PLS-DA, and OPLS-DA analysis (Figures 8B-D). Next, to identify key metabolites, we visualized the top 50 metabolites on a heatmap and identified nineteen metabolic pathways that were significantly differentially expressed between the $C$. albicans-infected mice and DADS-treated mice $(P<0.05$, Figures 8E, F). Additionally, we identified seventeen important metabolites for further analysis between the two groups (Table 3). All differentially expressed metabolites related to tryptophan metabolism were upregulated in the DADS group, including 4-(2-aminophenyl)-2,4-dioxobutanoic acid, kynurenic acid, N-acetylisatin, 5-hydroxyindoleacetic acid, quinoline-4,8diol, 4-(2-amino-3-hydroxyphenyl)-2,4-dioxobutanoic acid, 3methyldioxyindole, and 2-formaminobenzoylacetate. The levels of some metabolites, such as PGB2, PGD2, TXA2, and lipoxin A4, were increased in DADS-treated mice compared with $C$. albicans-infected mice. Moreover, some metabolites of bile secretion pathways were at a higher level in DADS-treated mice, including deoxycholic acid, lithocholic acid, chenodeoxycholic acid, and cholic acid.

\section{DISCUSSION}

Colonization and invasion of intestinal C. albicans are usually the prerequisites for disseminated $C$. albicans infection. To date, although many studies have reported that the gut microbiota, metabolites, and intestinal C. albicans could interact with each 
A

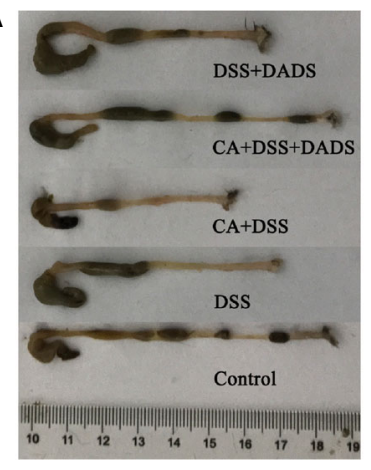

C

C Control
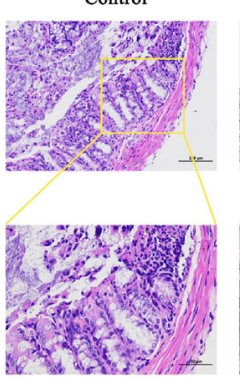

D

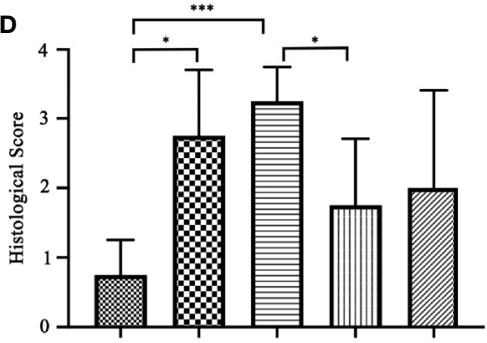

F

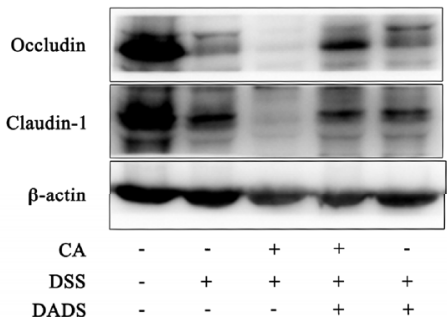

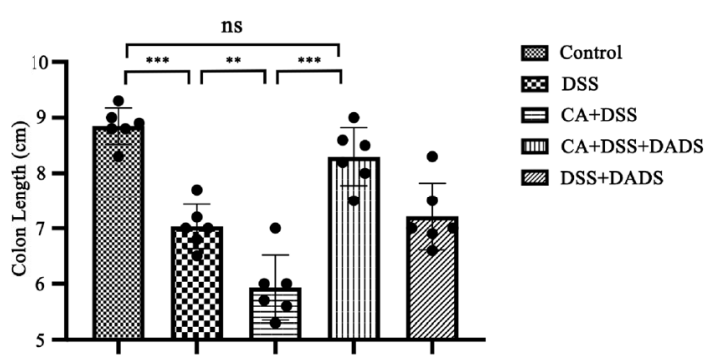

CA+DSS
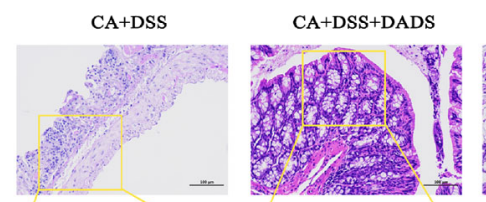

DSS+DADS
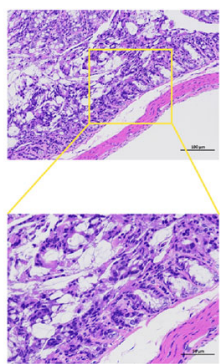

E

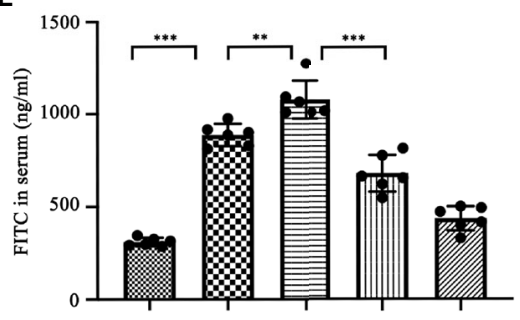

G

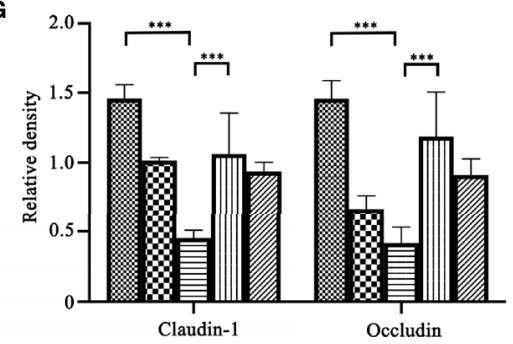

FIGURE 5 | DADS protected the epithelial barrier of mice with C. albicans infection. (A, B) Colon length and relevant statistical analysis. (C, D) H\&E staining of colon pathological changes and relevant scores. (E) FITC levels in the serum. (F, G) The levels of tight junction proteins, including Clauding-1 and Occludin. ${ }^{*}<0.05$, ${ }^{\star *} P<0.01$, and ${ }^{\star \star *} P<0.001$.

other, comprehensive research has been lacking, as the specific changes in bacteria and metabolites and regulatory mechanisms vary among animal models of intestinal C. albicans infection due to differences in intervention measures and other factors. This study mainly explored the effects of DADS, commonly known as allicin, on the gut microbiota, metabolites, and intestinal barrier of DSS-induced mice infected with enterogenic C. albicans. The results indicated that DADS could reduce the intestinal destruction caused by DSS and enterogenic C. albicans by increasing the expression of intestinal tight junction proteins, reducing intestinal inflammation (reflected by improvements in survival rate, weight change, colon length change, DAI score, and $\mathrm{H} \& \mathrm{E}$ scores) and reducing the expression of inflammatory factors in the serum and colon. In addition, DADS improved the gut microbiota and intestinal metabolite profiles of mice infected with intestinal C. albicans and increased the expression of beneficial bacteria and the expression of related bile acids and amino acids.

With regard to intestinal mucosal damage, C. albicans colonization of the gut could break through the intestinal 
TABLE 1 | The alpha diversity in each group of mice.

\begin{tabular}{|c|c|c|c|c|c|}
\hline Items & Control & DSS & CA + DSS & CA + DSS + DADS & DSS + DADS \\
\hline OTUs & $598.83 \pm 27.72$ & $462.67 \pm 41.47$ & $484.5 \pm 63.4$ & $470.83 \pm 33.09$ & $572.57 \pm 72.04$ \\
\hline Chao1 & $771.81 \pm 56.89$ & $677.52 \pm 42.86$ & $687.99 \pm 53.67$ & $642.59 \pm 43.3$ & $808.85 \pm 62.42$ \\
\hline Goods_coverage & $0.99502 \pm 0.00066$ & $0.9948 \pm 0.00027$ & $0.99486 \pm 0.00028$ & $0.99556 \pm 0.00042$ & $0.99445 \pm 0.00034$ \\
\hline Observed_species & $598.8 \pm 29.95$ & $459.1 \pm 38.36$ & $478.87 \pm 58.82$ & $468.18 \pm 29.91$ & $593.93 \pm 58.77$ \\
\hline Shannon & $5.43 \pm 0.49$ & $3.53 \pm 0.6$ & $4.8 \pm 0.54$ & $5.07 \pm 0.96$ & $5.9 \pm 0.3$ \\
\hline Simpson & $0.93 \pm 0.03$ & $0.75 \pm 0.12$ & $0.92 \pm 0.02$ & $0.9 \pm 0.07$ & $0.96 \pm 0.01$ \\
\hline
\end{tabular}

barrier to cause further gut tissue damage, disseminated $C$. albicans infection, and even the death of the host. While it is well recognized that $C$. albicans can induce inflammatory bowel dystrophy, the content of $C$. albicans in the intestines of mice is not as high as that in human intestines, and C. albicans cannot colonize the intestines of mice by oral-gastric gavage alone (Kumamoto, 2011; Koh, 2013; Hoarau et al., 2016). Therefore, the oral administration of C. albicans in the DSS-induced colitis mouse model may be a better model of the condition in humans. Consistent with previous reports, we observed significant weight loss, decreased survival, increased DAI scores, and greater colonization of C. albicans in mice with DSS-induced intestinal C. albicans infection (Medrano-Díaz et al., 2018; Hirayama et al., 2020). However, all of the above indicators were significantly improved by $20 \mathrm{mg} / \mathrm{kg}$ DADS intervention compared with the control treatment, which proved that DADS is effective in the treatment of intestinal C. albicans infection. Meanwhile, the intestinal barrier is one of the target organs that is destroyed by intestinal C. albicans and plays an important role in preventing disseminated C. albicans disease. We quantified the extent of intestinal destruction by testing the concentration of FITC-dextran in the serum in our experimental groups. FITCdextran is a fluorescent molecule that can cross a damaged intestinal barrier and be transepithelially transported into the blood. Therefore, the higher the content of fluorescent substances in the blood is, the more serious the destruction of the intestinal tract (Gupta and Nebreda, 2014). The most serious intestinal damage was observed in the CA + DSS group, which showed a significant difference from the DSS group. Moreover, these results indicated that $C$. albicans could aggravate intestinal destruction induced by DSS. In the CA + DSS + DADS group, we observed a significantly decreased concentration of FITCdextran, which showed that DADS ameliorated the effect of $C$. albicans infection on intestinal permeability. We further
A

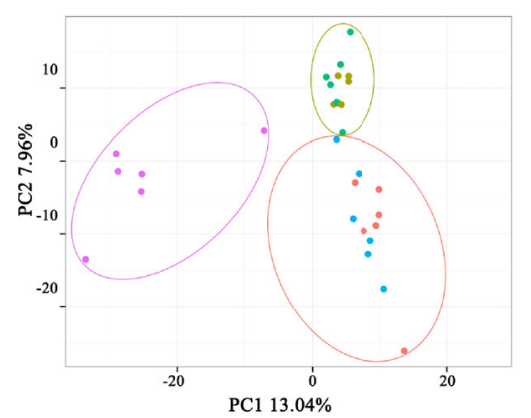

C

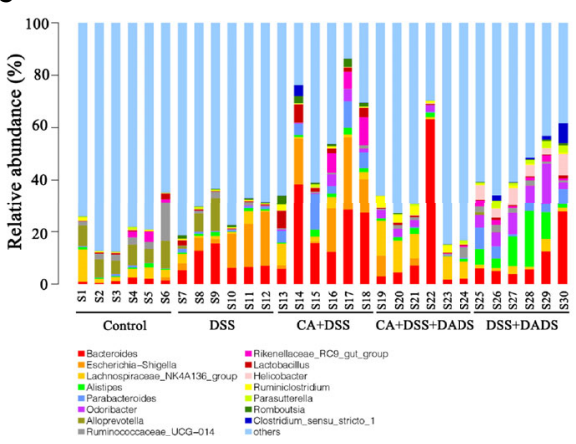

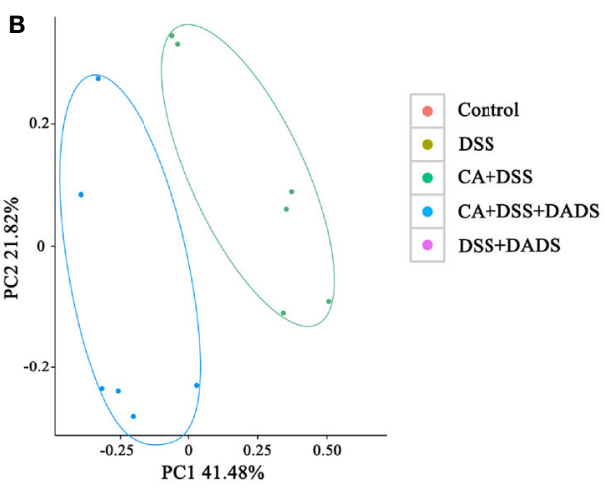

D

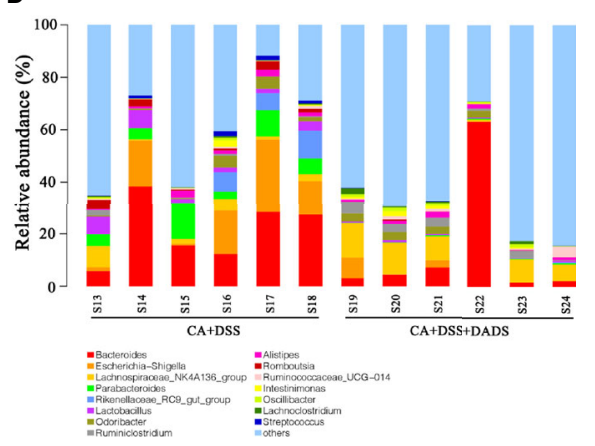

FIGURE 6 | DADS altered the gut microbiota community composition of mice with C. albicans infection. (A, B) Principal component analysis. (C, D) The relative abundances of the top 15 bacteria at the genus level among groups. ${ }^{*} P<0.05,{ }^{* *} P<0.01$. 
A

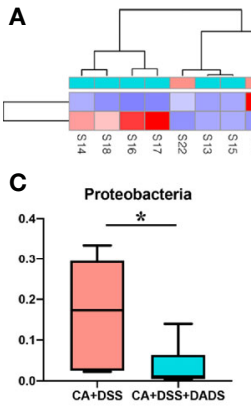

E Escherichia-Shigella

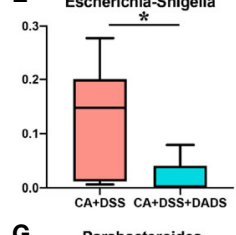

G Parabacteroides

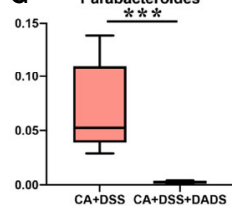

I Prevotellaceae_NK3B31_group

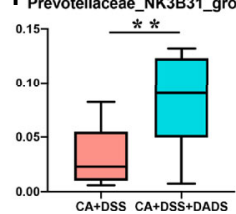

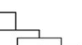

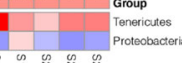

D
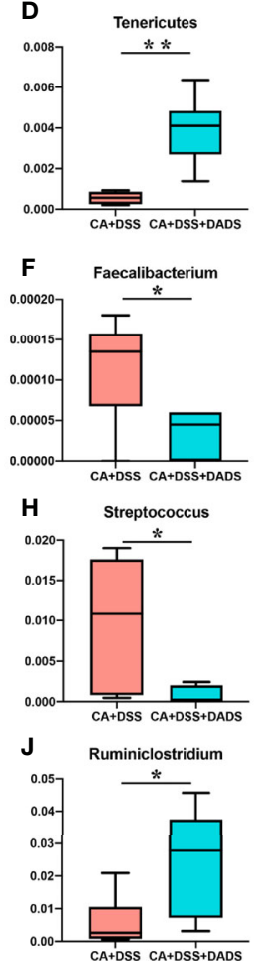
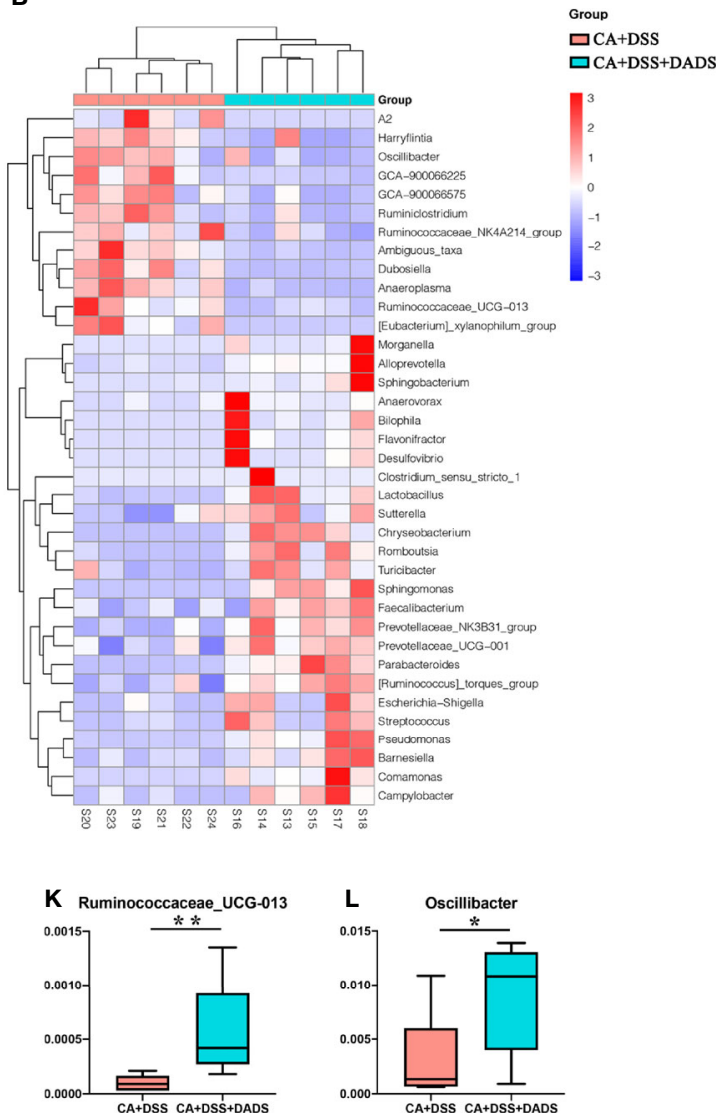

FIGURE 7 | Heatmaps of key bacteria at the (A) phylum and (B) genus levels. The relative abundances of (C) Proteobacteria, (D) Tenericutes, (E) EscherichiaShigella, (F) Faecalibacterium, (G) Parabacteroides, (H) Streptococcus, (I) Prevotellaceae_NK3B31_group, (J) Ruminiclostridium, (K) Ruminococcaceae_UCG-013, and (L) Oscillibacter in the two groups.

compared the intestinal barriers of each group based on intestinal pathology and the expression of intestinal tight junction proteins. In terms of intestinal pathology, colon length can be shortened by DSS-induced intestinal inflammation (Jawhara et al., 2012). We found that the CA +
DSS group had a shorter intestinal length and higher intestinal $\mathrm{H} \& \mathrm{E}$ pathological score than the DSS group, indicating that $C$. albicans further increased intestinal inflammation and destruction of the intestinal mucosa. In addition, the expression of intestinal tight junction proteins can reflect the

TABLE 2 | Changes of gut microbiota at different levels among the two groups.

\begin{tabular}{|c|c|c|c|c|c|}
\hline \multirow[t]{2}{*}{ Phylum/class } & \multirow[t]{2}{*}{ Family/genus } & \multicolumn{2}{|c|}{ Relative contribution ${ }^{a}$} & \multirow[t]{2}{*}{ Fold change $^{b}$} & \multirow[t]{2}{*}{ p-value ${ }^{c}$} \\
\hline & & CA+DSS & CA+DSS+DADS & & \\
\hline \multirow[t]{6}{*}{ Firmicutes } & - & $23.20 \%$ & $31.14 \%$ & 1.342660362 & 0.392 \\
\hline & Faecalibacterium & $0.01 \%$ & $0.0035 \%$ & 0.304347826 & 0.027 \\
\hline & Streptococcus & $0.99 \%$ & $0.08 \%$ & 0.083713851 & 0.023 \\
\hline & Ruminiclostridium & $0.58 \%$ & $2.46 \%$ & 4.263660017 & 0.023 \\
\hline & Ruminococcaceae_UCG-013 & $0.01 \%$ & $0.06 \%$ & 5.75 & 0.002 \\
\hline & Oscillibacter & $0.32 \%$ & $0.90 \%$ & $282.03 \%$ & 0.038 \\
\hline \multirow[t]{2}{*}{ Proteobacteria } & - & $16.85 \%$ & $3.46 \%$ & 0.205146491 & 0.019 \\
\hline & Escherichia-Shigella & $1.82 \%$ & $12.79 \%$ & 7.018131868 & 0.014 \\
\hline Tenericutes & - & $0.06 \%$ & $0.39 \%$ & 6.89380531 & 0.002 \\
\hline \multirow[t]{3}{*}{ Bacteroidetes } & - & $59.62 \%$ & $64.62 \%$ & 1.083814932 & 0.694 \\
\hline & Parabacteroides & $6.93 \%$ & $0.26 \%$ & 0.03698086 & 0.001 \\
\hline & Prevotellaceae_NK3B31_group & $0.01 \%$ & $0.0015 \%$ & 0.136363636 & 0.002 \\
\hline
\end{tabular}

${ }^{a}$ Relative contribution, ${ }^{b}$ Fold change, ${ }^{c} p$-value. 
A

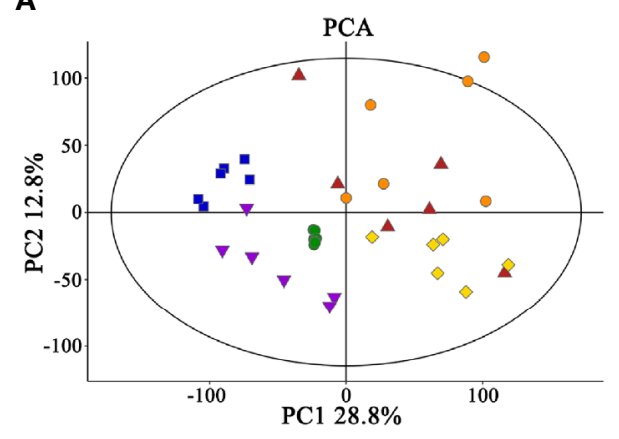

C

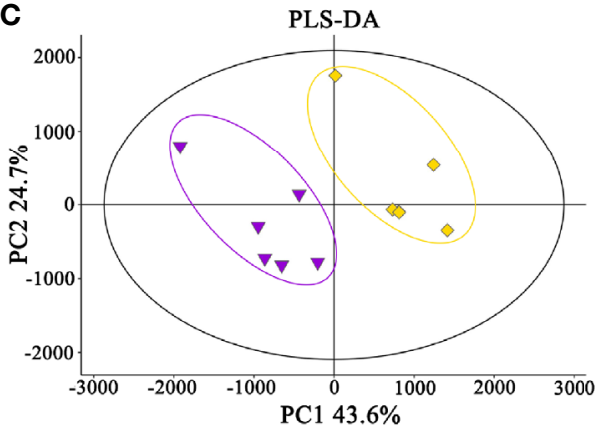

B

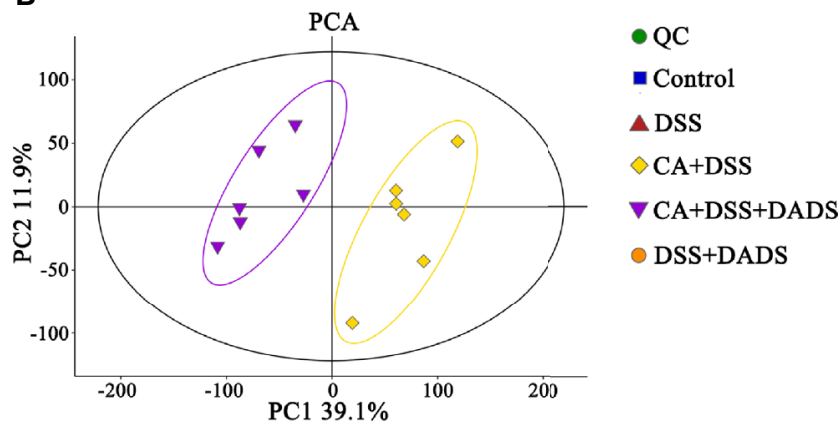

D

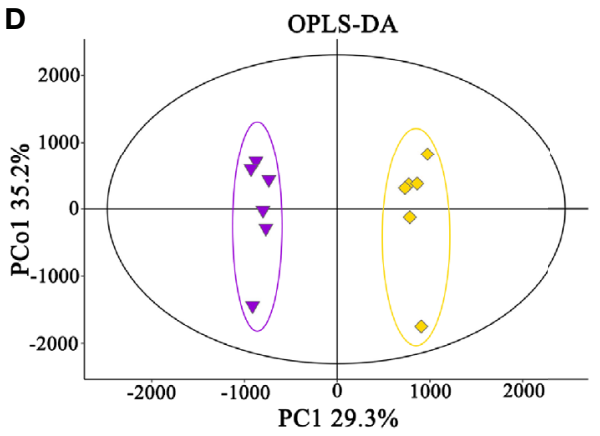

E

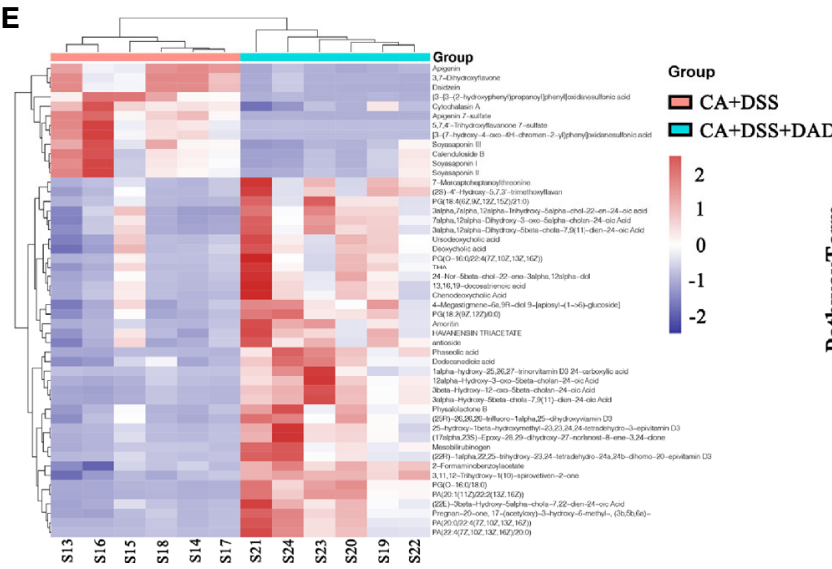

F

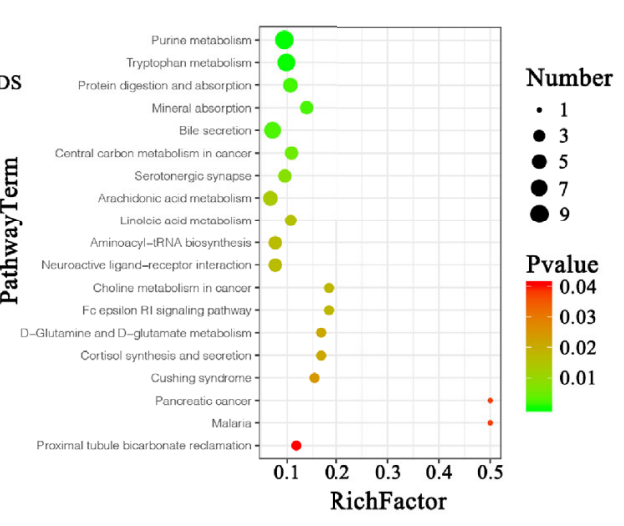

FIGURE 8 | Effect of DADS on fecal metabolites in mice with C. albicans infection. The (A, B) PCA, (C) PLS-DA, and (D) OPLS-DA models in different groups. (E) Heatmaps of differentially altered metabolites between the two groups. (F) Differential metabolic pathways visualized in bubble plots $(P<0.05)$; the bubble size represents the number of metabolites, $n=6$.

integrity of the intestinal mucosa at the protein level (Buckley and Turner, 2018; Shao et al., 2018). Claudin-1 and Occludin were hardly expressed in the CA + DSS group and were expressed at somewhat higher levels in the DSS group. However, in the DADS group, the expression of tight junction proteins was increased compared with that in the CA + DSS or DSS group, which indicated that DADS could increase the expression of colonic tight junction proteins and repair the damage to the intestinal mucosa. We also explored the condition of inflammation among groups by comparing the spleen index and the levels of IL- 6 and IFN- $\gamma$ in the serum, spleen and colon. We found that the spleen index and the expression of IL- 6 and IFN- $\gamma$ was decreased in the DADS group compared with the DSS and CA + DSS groups, but there was no significant difference among the groups in the expression of inflammatory factors in the spleen, which might be related to differences in expression in various tissues or depletion of these factors in the spleen. Compared with the DSS group, the CA + DSS group expressed more serious intestinal damage and inflammation, but DADS treatment alleviated gut damage and inflammation symptoms, which indicated that DADS could repair the intestinal damage caused by $C$. albicans by protecting gut tissues, increasing the expression of tight junction proteins, and reducing inflammation. 


\begin{tabular}{|c|c|c|c|c|c|c|c|c|c|}
\hline & & & & & & & & & \\
\hline & & & & CA + DSS & CA + DSS + DADS & $\mathrm{FC}^{\mathbf{b}}$ & P-value ${ }^{c}$ & & \\
\hline 1 & 4-(2-Aminophenyl)-2,4-dioxobutanoic acid & C01252 & 5.11 & $3006.75 \pm 774.77$ & $5,814.92 \pm 753.11$ & 1.93 & 0.0001 & Tryptophan metabolism & $\begin{array}{l}\text { Organooxygen } \\
\text { compounds }\end{array}$ \\
\hline 2 & Kynurenic acid & $\mathrm{C} 01717$ & 3.09 & $869.16 \pm 341.31$ & $1,893.54 \pm 202.69$ & 2.18 & 0.0001 & Tryptophan metabolism & $\begin{array}{c}\text { Quinolines and } \\
\text { derivatives }\end{array}$ \\
\hline 3 & $\mathrm{~N}$-Acetylisatin & C02172 & 2.27 & $764.47 \pm 178.48$ & $1,329.03 \pm 149.9$ & 1.74 & 0.0001 & Tryptophan metabolism & Unclassified \\
\hline 4 & 5-Hydroxyindoleacetic acid & C05635 & 3.19 & $842.47 \pm 275.36$ & $1,967.16 \pm 388.89$ & 2.33 & 0.0002 & Tryptophan metabolism|Serotonergic synapse & Indoles and derivatives \\
\hline 5 & Quinoline-4,8-diol & $\mathrm{C} 05637$ & 4.42 & $3028.48 \pm 694.56$ & $5,182.43 \pm 587.04$ & 1.71 & 0.0002 & Tryptophan metabolism & $\begin{array}{l}\text { Quinolines and } \\
\text { derivatives }\end{array}$ \\
\hline 6 & $\begin{array}{l}\text { 4-(2-Amino-3-hydroxyphenyl)-2,4- } \\
\text { dioxobutanoic acid }\end{array}$ & C05645 & 1.13 & $19.13 \pm 5.42$ & $167.84 \pm 91.38$ & 8.78 & 0.0026 & Tryptophan metabolism & $\begin{array}{l}\text { Organooxygen } \\
\text { compounds }\end{array}$ \\
\hline 7 & 3-Methyldioxyindole & C05834 & 1.16 & $209.32 \pm 84.2$ & $389.78 \pm 118.85$ & 1.86 & 0.0126 & Tryptophan metabolism & Indoles and derivatives \\
\hline 8 & 2-Formaminobenzoylacetate & C05835 & 8.52 & $10312.75 \pm 2153.38$ & $1,8075.57 \pm 1,919.96$ & 1.75 & 0.0001 & Tryptophan metabolism & Unclassified \\
\hline 9 & TXA2 & C02198 & 1.22 & $30.74 \pm 9.6$ & $178.88 \pm 37.47$ & 5.82 & 0.0000 & Serotonergic synapse & Fatty Acyls \\
\hline 10 & PGB2 & C05954 & 1.21 & $65.36 \pm 29.6$ & $224.88 \pm 70.48$ & 3.44 & 0.0005 & Serotonergic synapse & Fatty Acyls \\
\hline 11 & PGD2 & C00696 & 1.12 & $6.21 \pm 4.59$ & $203.97 \pm 210.74$ & 32.87 & 0.0444 & Serotonergic synapse & Fatty Acyls \\
\hline 12 & Lipoxin A4 & C06314 & 1.75 & $521.63 \pm 208.05$ & $891.74 \pm 97.67$ & 1.71 & 0.0028 & Serotonergic synapse & Fatty Acyls \\
\hline 13 & Deoxycholic acid & $\mathrm{C} 04483$ & 6.13 & $5,889.56 \pm 4,018.49$ & $1,1702.38 \pm 4,298.96$ & 1.99 & 0.0361 & Bile secretion & $\begin{array}{l}\text { Bile acids and } \\
\text { derivatives }\end{array}$ \\
\hline 14 & Lithocholic acid & C03990 & 3.09 & $269 \pm 119.43$ & $1,650.66 \pm 1,261.03$ & 6.14 & 0.0234 & Bile secretion & $\begin{array}{l}\text { Bile acids and } \\
\text { derivatives }\end{array}$ \\
\hline 15 & Lithocholate 3-O-glucuronide & C03033 & 1.38 & $150.05 \pm 92.62$ & $426.1 \pm 200.56$ & 2.84 & 0.0120 & $\begin{array}{l}\text { Bile secretion|Pentose and glucuronate } \\
\text { interconversions }\end{array}$ & Steroidal glycosides \\
\hline 16 & Chenodeoxycholic Acid & $\mathrm{C} 02528$ & 7.61 & $2,870.1 \pm 3,115$ & $1,1744.1 \pm 8,335.01$ & 4.09 & 0.0347 & Bile secretion|Primary bile acid biosynthesis & $\begin{array}{l}\text { Bile acids and } \\
\text { derivatives }\end{array}$ \\
\hline 17 & Cholic acid & C00695 & 3.65 & $1,932.38 \pm 998.97$ & $3,691.59 \pm 1,072.31$ & 1.91 & 0.0148 & Bile secretion|Primary bile acid biosynthesis & $\begin{array}{l}\text { Bile acids and } \\
\text { derivatives }\end{array}$ \\
\hline
\end{tabular}

${ }^{a}$ Relative contribution, ${ }^{b}$ Fold change, ${ }^{c} p$-value. 
Nowadays, nosocomial bloodstream infections caused by $C$. albicans rank third (Wisplinghoff et al., 2004). Moreover, the $C$. albicans in the blood mainly comes from the intestine (Kullberg and Arendrup, 2015). So the destruction of the intestines is conducive to the colonization, invasion, and infection of $C$. albicans. DADS, as a classic garlic active substance, its antifungal and digestive system protection effects have been confirmed, which is consistent with our above research (Shang et al., 2019). Few people study the association between gut protection and antifungal effect. The intestinal protective effect of DADS may reduce the colonization of C. albicans, thereby inhibiting the bloodstream entry of $C$. albicans from the intestine. Secondly, the antifungal effect of DADS reduces the destruction of $C$. albican to the intestine, which may also improve the intestinal barrier. But the detailed interaction mechanism needs further study. In addition, the role of DADS on gut microbiota and metabolites is also important for intestinal protection and anti-fungal.

The community of gut microbiota in the CA + DSS group was also significantly different from that in the healthy control group and the DSS group. Interestingly, similar to what was found in previous research, the abundance of Bacteroides, Bacteroidaceae, Proteobacteria, Escherichia-Shigella, Streptococcus, and other pathogenic bacteria was increased in mice with DSS-induced intestinal C. albicans infection. It is possible that the increased mortality in the CA + DSS group was related to bacteremia caused by pathogenic bacteria that selectively break through the intestinal barrier (Hiengrach et al., 2019). Bacteremia may also cause high levels of inflammation in the serum and intestines and increased levels of inflammatory factors (IL-6, IFN- $\gamma$ ) (Michielan and D'incà, 2015). Proteobacteria are typically highly abundant in certain intestinal and extraintestinal diseases with inflammatory manifestations, so it is also considered to be a possible microbial feature of these diseases (Rizzatti et al., 2017). In this study, we found that the abundances of Proteobacteria (at the phylum level) and Escherichia-Shigella (at the genus level) were increased in the CA + DSS group. Studies have shown that Proteobacteria could be a biomarker indicating the instability of the gut microbiota, which can invade intestinal epithelial cells and aggravate intestinal inflammation by releasing endotoxins and lipopolysaccharide (LPS) (Boudeau et al., 1999; Lu and Walker, 2001; Belotserkovsky and Sansonetti, 2018). Consistent with previous experiments using similar mouse models with oral-gastric gavage of C. albicans, the CA + DSS group exhibited significant enrichment of Bacteroides, which is usually a commensal bacterium in the host intestine but may become a pathogen under certain conditions. Previous studies have reported that Bacteroides can produce LPS and a variety of enzymes, thereby enhancing the adhesion of bacteria to the intestinal tissues of the host and protecting them from immune attack, ultimately leading to the destruction of the intestinal epithelium (Wexler, 2007; Sears, 2009). In addition, Bacteroides can produce enterotoxin to cleave tight junction proteins in the intestine, leading to cytoskeletal rearrangement and loss of tight junctions in the intestinal epithelial cells, which may explain the decreased expression of Claudin-1 and Occludin
(Wu et al., 1998). In the CA + DSS + DADS group, we observed an increased abundance of Ruminococcaceae_UCG-013, Ruminococcaceae_NK4A214_group, Ruminiclostridium, and Oscillibacter, which are usually not abundant in patients with ulcerative colitis. These results might indicate that DADS can increase the abundance of beneficial bacteria such as Ruminococcus to reduce intestinal inflammation (Hyams et al., 2019). In addition, Ruminiclostridium has been reported to be increased in healthy mice and to produce butyric acid, which can nourish and protect the intestinal epithelium (Machiels et al., 2014). Thus, these findings indicated that DADS treatment might mitigate intestinal C. albicans infection by improving the disordered gut microbiota.

Meanwhile, the intestinal metabolite homeostasis was also altered among the groups. Gut metabolites are closely related to some intestinal and extraintestinal diseases, such as inflammatory bowel disease, irritable bowel syndrome, depression, and autism (Duboc et al., 2012; Bjerrum et al., 2015; Sharon et al., 2019; Keshteli et al., 2019). Moreover, some gut metabolites could protect or destroy the gut barrier (Li et al., 2019; Parada Venegas et al., 2019). In this study, the intestinal metabolite profiles were significantly different between the CA + DSS and CA + DSS + DADS groups. In addition, through KEGG data analysis, we found 19 different metabolic pathways. In terms of the bile acid secretion metabolism, the content of bile acids, such as deoxycholic acid, chenodeoxycholic acid, ursodeoxycholic acid, lithocholic acid, and cholic acid, was significantly higher in the CA + DSS + DADS group than in the CA + DSS group, similar to what has been found in patients with inflammatory bowel disease (Jansson et al., 2009; Duboc et al., 2013). Deoxycholic acid could inhibit the secretion of IL-1 $\beta$ and IL- 8 by intestinal epithelial Caco- 2 cells in a dose-dependent manner (Yao et al., 2019; Lavelle and Sokol, 2020). The metabolites involved in arachidonic acid metabolism, such as TXA2, PGD2, and PGB2, were significantly increased in the CA + DSS + DADS group. Studies have shown that arachidonic acid metabolites could increase the sensitivity of $C$. albicans to fluconazole (Kuloyo et al., 2020). All metabolites involved in the tryptophan metabolic pathway were enriched in the CA + DSS + DADS group and were significantly higher than in the CA + DSS group. Research has proven that tryptophan metabolism plays a key role in regulating the immune response of the body against $C$. albicans. Tryptophan could activate the microbialdependent AhR/IL-22 axis to inhibit fungal growth and infection on the mucosal surface and thus prevent abnormal immune stimulation by C. albicans (Romani et al., 2008). In addition, the interaction between tryptophan metabolites and C. albicans may reduce the toxicity of the fungus (Mayr et al., 2005). Activation of the tryptophan metabolic pathway can produce bioactive molecules to maintain the homeostasis of the intestinal mucosa, which makes tryptophan metabolites the main players in intestinal health (Renga et al., 2019). Therefore, these findings indicated that DADS may inhibit the invasion of intestinal $C$. albicans and ameliorate intestinal barrier damage by activating specific metabolic pathways and increasing the levels of related metabolites. 


\section{CONCLUSION}

In this study, we established DSS-induced intestinal C. albicans mouse models to better simulate human intestinal fungal infections. We found that C. albicans could enhance DSS colitis severity and that DADS treatment could improve intestinal dysbiosis (altered gut microbiota and metabolites), gut permeability and systemic inflammatory responses. Insight into the effects of DADS treatment may provide novel treatment strategies for intestinal C. albicans infection.

\section{DATA AVAILABILITY STATEMENT}

The original contributions presented in the study are publicly available. This data can be found in NCBI under accession number PRJNA778766, and at MetaboLights under accession number MTBLS3750.

\section{ETHICS STATEMENT}

The animal study was reviewed and approved by the Experimental Animal Ethical Review Committee, East China Normal University.

\section{REFERENCES}

Alam, M., Zubair, S., Farazuddin, M., Ahmad, E., Khan, A., Zia, Q., et al. (2013). Development, Characterization and Efficacy of Niosomal Diallyl Disulfide in Treatment of Disseminated Murine Candidiasis. Nanomedicine 9 (2), 247-256. doi: 10.1016/j.nano.2012.07.004

Basmaciyan, L., Bon, F., Paradis, T., Lapaquette, P., and Dalle., F. (2019). “Candida Albicans Interactions With The Host: Crossing The Intestinal Epithelial Barrier”. Tissue Barriers 7 (2), 1612661. doi: 10.1080/21688370.2019.1612661

Belotserkovsky, I., and Sansonetti, P. J. (2018). Shigella and Enteroinvasive Escherichia Coli. Curr. Top. Microbiol. Immunol. 416, 1-26. doi: 10.1007/ 82_2018_104

Benavides, G. A., Squadrito, G. L., Mills, R. W., Patel, H. D., Isbell, T. S., Patel, R. P., et al. (2007). Hydrogen Sulfide Mediates the Vasoactivity of Garlic. Proc. Natl. Acad. Sci. U.S.A. 104 (46), 17977-17982. doi: 10.1073/pnas.0705710104

Bertolini, M., Ranjan, A., Thompson, A., Diaz, P. I., Sobue, T., Maas, K., et al. (2019). Candida Albicans Induces Mucosal Bacterial Dysbiosis That Promotes Invasive Infection. PloS Pathog. 15 (4), e1007717. doi: 10.1371/ journal.ppat.1007717

Bessede, A., Gargaro, M., Pallotta, M. T., Matino, D., Servillo, G., Brunacci, C., et al. (2014). Aryl Hydrocarbon Receptor Control of a Disease Tolerance Defence Pathway. Nature 511 (7508), 184-190. doi: 10.1038/nature13323

Bjerrum, J. T., Wang, Y., Hao, F., Coskun, M., Ludwig, C., Günther, U., et al. (2015). Metabonomics of Human Fecal Extracts Characterize Ulcerative Colitis, Crohn's Disease and Healthy Individuals. Metabolomics 11, 122-133. doi: 10.1007/s11306-014-0677-3

Bongomin, F., Gago, S., Oladele, R. O., and Denning., D. W. (2017). Global and Multi-National Prevalence of Fungal Diseases-Estimate Precision. J. Fungi (Basel) 3 (4), 57. doi: 10.3390/jof3040057

Boudeau, J., Glasser, A. L., Masseret, E., Joly, B., and Darfeuille-Michaud., A. (1999). Invasive Ability of an Escherichia Coli Strain Isolated From the Ileal Mucosa of a Patient With Crohn's Disease. Infect. Immun. 67 (9), 4499-4509. doi: 10.1128/IAI.67.9.4499-4509.1999

Buckley, A., and Turner, J. R. (2018). Cell Biology of Tight Junction Barrier Regulation and Mucosal Disease. Cold Spring Harb. Perspect. Biol. 10 (1), a029314. doi: 10.1101/cshperspect.a029314

\section{AUTHOR CONTRIBUTIONS}

WH: Conceptualization, Methodology, Investigation, Formal analysis, Writing-Original Draft, Visualization. LH: Conceptualization, Software, Resources. ZZ: Data Curation, Formal analysis. LY: Investigation, data collection, Fecal samples collection, Resources. JT: Conceptualization, Methodology, Writing-Review \& Editing, Supervision. All authors contributed to the article and approved the submitted version.

\section{FUNDING}

This study was funded by the Fifth People's Hospital of Shanghai, Fudan University (grant number 2018WYZD03, 2020WYZDZK06) and the Shanghai Science and Technology Commission (grant number 18411950600).

\section{ACKNOWLEDGMENTS}

We are grateful for the support and helpful assistance from the Central Laboratory, the Fifth People's Hospital of Shanghai, Fudan University and the China Scholarship Council.

Caporaso, J. G., Bittinger, K., Bushman, F. D., DeSantis, T. Z., Andersen, G. L., and Knight., R. (2010). PyNAST: A Flexible Tool for Aligning Sequences to a Template Alignment. Bioinformatics 26 (2), 266-267. doi: 10.1093/ bioinformatics/btp636

Christophi, G. P., Rengarajan, A., and Ciorba, M. A. (2016). Rectal Budesonide and Mesalamine Formulations in Active Ulcerative Proctosigmoiditis: Efficacy, Tolerance, and Treatment Approach. Clin. Exp. Gastroenterol. 9, 125-130. doi: $10.2147 /$ ceg.S80237

Duboc, H., Rainteau, D., Rajca, S., Humbert, L., Farabos, D., Maubert, M., et al. (2012). Increase in Fecal Primary Bile Acids and Dysbiosis in Patients With Diarrhea-Predominant Irritable Bowel Syndrome. Neurogastroenterol. Motil. 24 (6), 513-20, e246-7. doi: 10.1111/j.1365-2982.2012.01893.x

Duboc, H., Rajca, S., Rainteau, D., Benarous, D., Maubert, M. A., Quervain, E., et al. (2013). Connecting Dysbiosis, Bile-Acid Dysmetabolism and Gut Inflammation in Inflammatory Bowel Diseases. Gut 62 (4), 531-539. doi: 10.1136/gutjnl-2012-302578

Franzosa, E. A., Sirota-Madi, A., Avila-Pacheco, J., Fornelos, N., Haiser, H. J., Reinker, S., et al. (2019). Gut Microbiome Structure and Metabolic Activity in Inflammatory Bowel Disease. Nat. Microbiol. 4 (2), 293-305. doi: 10.1038/ s41564-018-0306-4

Gentile, C. L., and Weir, T. L. (2018). The Gut Microbiota at the Intersection of Diet and Human Health. Sci. (New York NY) 362 (6416), 776-780. doi: $10.1126 /$ science.aau5812

Gupta, J., and Nebreda, A. (2014). Analysis of Intestinal Permeability in Mice. BIO-PROTOCOL 4 (22), e1289. doi: 10.21769/BioProtoc.1289

Gutierrez, D., Weinstock, A., Antharam, V. C., Gu, H., Jasbi, P., Shi, X., et al. (2020). Antibiotic-Induced Gut Metabolome and Microbiome Alterations Increase the Susceptibility to Candida Albicans Colonization in the Gastrointestinal Tract. FEMS Microbiol. Ecol. 96 (1), fiz187. doi: 10.1093/femsec/fiz187

Heath-Pagliuso, S., Rogers, W. J., Tullis, K., Seidel, S. D., Cenijn, P. H., Brouwer, A., et al. (1998). Activation of the Ah Receptor by Tryptophan and Tryptophan Metabolites. Biochemistry 37 (33), 11508-11515. doi: 10.1021/bi980087p

He, H., Ma, Y., Huang, H., Huang, C., Chen, Z., Chen, D., et al. (2021). A Comprehensive Understanding About the Pharmacological Effect of Diallyl Disulfide Other Than its Anti-Carcinogenic Activities. Eur. J. Pharmacol. 893, 173803. doi: 10.1016/j.ejphar.2020.173803 
Hiengrach, P., Panpetch, W., Worasilchai, N., Chindamporn, A., Tumwasorn, S., Jaroonwitchawan, T., et al. (2019). Administration of Candida Albicans to Dextran Sulfate Solution Treated Mice Causes Intestinal Dysbiosis, Emergence and Dissemination of Intestinal Pseudomonas Aeruginosa and Lethal Sepsis. Shock (Augusta Ga) 53 (2), 189-198. doi: 10.1097/shk.0000000000001339

Hirayama, T., Miyazaki, T., Ito, Y., Wakayama, M., Shibuya, K., Yamashita, K., et al. (2020). Virulence Assessment of Six Major Pathogenic Candida Species in the Mouse Model of Invasive Candidiasis Caused by Fungal Translocation. Sci. Rep. 10 (1), 3814. doi: 10.1038/s41598-020-60792-y

Hoarau, G., Mukherjee, P. K., Gower-Rousseau, C., Hager, C., Chandra, J., Retuerto, M. A., et al. (2016). Bacteriome and Mycobiome Interactions Underscore Microbial Dysbiosis in Familial Crohn's Disease. mBio 7 (5), e01250-e01216. doi: 10.1128/mBio.01250-16

Hu, W., Xu, D., Zhou, Z., Zhu, J., Wang, D., and Tang., J. (2021). Alterations in the Gut Microbiota and Metabolic Profiles Coincide With Intestinal Damage in Mice With a Bloodborne Candida Albicans Infection. Microbial. Pathogenesis 154, 104826. doi: 10.1016/j.micpath.2021.104826

Hyams, J. S., Davis Thomas, S., Gotman, N., Haberman, Y., Karns, R., Schirmer, M., et al. (2019). Clinical and Biological Predictors of Response to Standardised Paediatric Colitis Therapy (PROTECT): A Multicentre Inception Cohort Study. Lancet 393 (10182), 1708-1720. doi: 10.1016/s0140-6736(18)32592-3

Imhann, F., Vich Vila, A., Bonder, M. J., Fu, J., Gevers, D., Visschedijk, M. C., et al. (2018). Interplay of Host Genetics and Gut Microbiota Underlying the Onset and Clinical Presentation of Inflammatory Bowel Disease. Gut 67 (1), 108-119. doi: 10.1136/gutjnl-2016-312135

Jansson, J., Willing, B., Lucio, M., Fekete, A., Dicksved, J., Halfvarson, J., et al. (2009). Metabolomics Reveals Metabolic Biomarkers of Crohn's Disease. PloS One 4 (7), e6386. doi: 10.1371/journal.pone.0006386

Jawhara, S., Mogensen, E., Maggiotto, F., Fradin, C., Sarazin, A., Dubuquoy, L., et al. (2012). Murine Model of Dextran Sulfate Sodium-Induced Colitis Reveals Candida Glabrata Virulence and Contribution of Beta-Mannosyltransferases. J. Biol. Chem. 287 (14), 11313-11324. doi: 10.1074/jbc.M111.329300

Keshteli, A. H., Madsen, K. L., Mandal, R., Boeckxstaens, G. E., Bercik, P., De Palma, G., et al. (2019). Comparison of the Metabolomic Profiles of Irritable Bowel Syndrome Patients With Ulcerative Colitis Patients and Healthy Controls: New Insights Into Pathophysiology and Potential Biomarkers. Aliment Pharmacol. Ther. 49 (6), 723-732. doi: 10.1111/apt.15141

Khodavandi, A., Harmal, N. S., Alizadeh, F., Scully, O. J., Sidik, S. M., Othman, F., et al. (2011). Comparison Between Allicin and Fluconazole in Candida Albicans Biofilm Inhibition and in Suppression of HWP1 Gene Expression. Phytomed.: Int. J. Phytother. Phytopharmacol. 19 (1), 56-63. doi: 10.1016/ j.phymed.2011.08.060

Koh, A. Y. (2013). Murine Models of Candida Gastrointestinal Colonization and Dissemination. Eukaryot. Cell 12 (11), 1416-1422. doi: 10.1128/ec.00196-13

Koh, A. Y., Köhler, J. R., Coggshall, K. T., Van Rooijen, N., and Pier., G. B. (2008). Mucosal Damage and Neutropenia are Required for Candida Albicans Dissemination. PloS Pathog. 4 (2), e35. doi: 10.1371/journal.ppat.0040035

Kullberg, B. J., and Arendrup, M. C. (2015). Invasive Candidiasis. N Engl. J. Med. 373 (15), 1445-1456. doi: 10.1056/NEJMra1315399

Kuloyo, O., Fourie, R., Cason, E., Albertyn, J., and Pohl., C. H. (2020). Transcriptome Analyses of Candida Albicans Biofilms, Exposed to Arachidonic Acid and Fluconazole, Indicates Potential Drug Targets. G3 (Bethesda) 10 (9), 3099-3108. doi: 10.1534/g3.120.401340

Kumamoto, C. A. (2011). Inflammation and Gastrointestinal Candida Colonization. Curr. Opin. Microbiol. 14 (4), 386-391. doi: 10.1016/j.mib.2011.07.015

Kumamoto, C. A., Gresnigt, M. S., and Hube, B. (2020). The Gut, the Bad and the Harmless: Candida Albicans as a Commensal and Opportunistic Pathogen in the Intestine. Curr. Opin. Microbiol. 56, 7-15. doi: 10.1016/j.mib.2020.05.006

Lanis, J. M., Kao, D. J., Alexeev, E. E., and Colgan., S. P. (2017). Tissue Metabolism and the Inflammatory Bowel Diseases. J. Mol. Med. (Berl.) 95 (9), 905-913. doi: 10.1007/s00109-017-1544-2

Lavelle, A., and Sokol, H. (2020). Gut Microbiota-Derived Metabolites as Key Actors in Inflammatory Bowel Disease. Nat. Rev. Gastroenterol. Hepatol. 17 (4), 223-237. doi: 10.1038/s41575-019-0258-z

Lemar, K. M., Passa, O., Aon, M. A., Cortassa, S., Muller, C. T., Plummer, S., et al. (2005). Allyl Alcohol and Garlic (Allium Sativum) Extract Produce Oxidative Stress in Candida Albicans. Microbiol. (Read. England) 151 (10), 3257-3265. doi: $10.1099 /$ mic.0.28095-0
Liang, D., Wu, H., Wong, M. W., and Huang, D. (2015). Diallyl Trisulfide Is a Fast H2S Donor, But Diallyl Disulfide Is a Slow One: The Reaction Pathways and Intermediates of Glutathione With Polysulfides. Org. Lett. 17 (17), 4196-4199. doi: 10.1021/acs.orglett.5b01962

Li, Z., Li, J., Zhang, S., Chen, G., Chi, S., Li, X., et al. (2019). Metabolomics Analysis of Gut Barrier Dysfunction in a Trauma-Hemorrhagic Shock Rat Model. Biosci. Rep. 39 (1), BSR20181215. doi: 10.1042/bsr20181215

Liu, Z., Liu, F., Li, G., Chi, X., Wang, Y., Wang, H., et al. (2020). Metabolite Support of Long-Term Storage of Sperm in the Spermatheca of Honeybee (Apis Mellifera) Queens. Front. Physiol. 11, 574856. doi: 10.3389/ fphys.2020.574856

Lu, L., and Walker, W. A. (2001). Pathologic and Physiologic Interactions of Bacteria With the Gastrointestinal Epithelium. Am. J. Clin. Nutr. 73 (6), 1124s1130s. doi: 10.1093/ajcn/73.6.1124S

Machiels, K., Joossens, M., Sabino, J., De Preter, V., Arijs, I., Eeckhaut, V., et al. (2014). A Decrease of the Butyrate-Producing Species Roseburia Hominis and Faecalibacterium Prausnitzii Defines Dysbiosis in Patients With Ulcerative Colitis. Gut 63 (8), 1275-1283. doi: 10.1136/gutjnl-2013-304833

Mason, K. L., Erb Downward, J. R., Mason, K. D., Falkowski, N. R., Eaton, K. A., Kao, J. Y., et al. (2012). Candida Albicans and Bacterial Microbiota Interactions in the Cecum During Recolonization Following Broad-Spectrum Antibiotic Therapy. Infect. Immun. 80 (10), 3371-3380. doi: 10.1128/iai.00449-12

Mayr, A., Hinterberger, G., Dierich, M. P., and Lass-Flörl., C. (2005). Interaction of Serotonin With Candida Albicans Selectively Attenuates Fungal Virulence In Vitro. Int. J. Antimicrobial. Agents 26 (4), 335-337. doi: 10.1016/ j.ijantimicag.2005.07.006

Medrano-Díaz, C. L., Vega-González, A., Ruiz-Baca, E., Moreno, A., and CuéllarCruz., M. (2018). Moonlighting Proteins Induce Protection in a Mouse Model Against Candida Species. Microbial. Pathogenesis 124, 21-29. doi: 10.1016/ j.micpath.2018.08.024

Michielan, A., and D'incà, R. (2015). Intestinal Permeability in Inflammatory Bowel Disease: Pathogenesis, Clinical Evaluation, and Therapy of Leaky Gut. Mediators Inflamm. 2015, (628157). doi: 10.1155/2015/628157

Motta, J. P., Flannigan, K. L., Agbor, T. A., Beatty, J. K., Blackler, R. W., Workentine, M. L., et al. (2015). Hydrogen Sulfide Protects From Colitis and Restores Intestinal Microbiota Biofilm and Mucus Production. Inflamm. Bowel Dis. 21 (5), 1006-1017. doi: 10.1097/mib.0000000000000345

Neville, B. A., D’enfert, C., and Bougnoux, M. E. (2015). Candida Albicans Commensalism in the Gastrointestinal Tract. FEMS Yeast Res. 15 (7), fov081. doi: 10.1093/femsyr/fov081

Pappas, P. G., Lionakis, M. S., Arendrup, M. C., Ostrosky-Zeichner, L., and Kullberg., B. J. (2018). Invasive Candidiasis. Nat. Rev. Dis. Primers 4, 18026. doi: $10.1038 /$ nrdp.2018.26

Parada Venegas, D., de la Fuente, M. K., Landskron, G., González, M. J., Quera, R., Dijkstra, G., et al. (2019). Short Chain Fatty Acids (SCFAs)-Mediated Gut Epithelial and Immune Regulation and Its Relevance for Inflammatory Bowel Diseases. Front. Immunol. 10, 277. doi: 10.3389/fimmu.2019.00277

Renga, G., Bellet, M. M., Stincardini, C., Pariano, M., Oikonomou, V., Villella, V. R., et al. (2019). To Be or Not to Be a Pathogen: Candida Albicans and Celiac Disease. Front. Immunol. 10, 2844. doi: 10.3389/fimmu.2019.02844

Rizzatti, G., Lopetuso, L. R., Gibiino, G., Binda, C., and Gasbarrini., A. (2017). Proteobacteria: A Common Factor in Human Diseases. BioMed. Res. Int. 2017, (9351507). doi: 10.1155/2017/9351507

Rognes, T., Flouri, T., Nichols, B., Quince, C., and Mahé., F. (2016). VSEARCH: A Versatile Open Source Tool for Metagenomics. PeerJ 4, e2584. doi: 10.7717/ peerj. 2584

Romani, L., Zelante, T., De Luca, A., Fallarino, F., and Puccetti., P. (2008). IL-17 and Therapeutic Kynurenines in Pathogenic Inflammation to Fungi. J. Immunol. 180 (8), 5157-5162. doi: 10.4049/jimmunol.180.8.5157

Romani, L., Zelante, T., Palmieri, M., Napolioni, V., Picciolini, M., Velardi, A., et al. (2015). The Cross-Talk Between Opportunistic Fungi and the Mammalian Host via Microbiota's Metabolism. Semin. Immunopathol. 37 (2), 163-171. doi: 10.1007/s00281-014-0464-2

Sears, C. L. (2009). Enterotoxigenic Bacteroides Fragilis: A Rogue Among Symbiotes. Clin. Microbiol. Rev. 22 (2), 349-369. doi: 10.1128/cmr.00053-08

Shang, A., Cao, S. Y., Xu, X. Y., Gan, R. Y., Tang, G. Y., Corke, H., et al. (2019). Bioactive Compounds and Biological Functions of Garlic (Allium Sativum L.). Foods 8 (7), 246. doi: 10.3390/foods 8070246 
Shao, T., Zhao, C., Li, F., Gu, Z., Liu, L., Zhang, L., et al. (2018). Intestinal HIF-1 $\alpha$ Deletion Exacerbates Alcoholic Liver Disease by Inducing Intestinal Dysbiosis and Barrier Dysfunction. J. Hepatol. 69 (4), 886-895. doi: 10.1016/ j.jhep.2018.05.021

Sharon, G., Cruz, N. J., Kang, D. W., Gandal, M. J., Wang, B., Kim, Y. M., et al. (2019). Human Gut Microbiota From Autism Spectrum Disorder Promote Behavioral Symptoms in Mice. Cell 177 (6), 1600-1618.e17. doi: 10.1016/ j.cell.2019.05.004

Staniszewska, M. (2020). Virulence Factors in Candida Species. Curr. Protein Pept. Sci. 21 (3), 313-323. doi: 10.2174/1389203720666190722152415

Sun, M., Wu, W., Liu, Z., and Cong., Y. (2017). Microbiota Metabolite Short Chain Fatty Acids, GPCR, and Inflammatory Bowel Diseases. J. Gastroenterol. 52 (1), 1-8. doi: 10.1007/s00535-016-1242-9

Toda, M., Williams, S. R., Berkow, E. L., Farley, M. M., Harrison, L. H., Bonner, L., et al. (2019). Population-Based Active Surveillance for Culture-Confirmed Candidemia - Four Sites, United States, 2012-2016. MMWR Surveill. Summ. 68 (8), 1-15. doi: 10.15585/mmwr.ss6808al

Valentine, M., Benadé, E., Mouton, M., Khan, W., and Botha., A. (2019). Binary Interactions Between the Yeast Candida Albicans and Two Gut-Associated Bacteroides Species. Microbial. Pathogenesis 135, 103619. doi: 10.1016/ j.micpath.2019.103619

Watson, C., Mahe, M., and Helmrath, M. (2015). In Vivo Fluorescein Isothiocyanate-Dextran (FD4) Permeability Assay. Bio-Protocol 5 (20), e1618. doi: 10.21769/BioProtoc.1618

Wexler, H. M. (2007). Bacteroides: The Good, the Bad, and the Nitty-Gritty. Clin. Microbiol. Rev. 20 (4), 593-621. doi: 10.1128/cmr.00008-07

Wikoff, W. R., Anfora, A. T., Liu, J., Schultz, P. G., Lesley, S. A., Peters, E. C., et al. (2009). Metabolomics Analysis Reveals Large Effects of Gut Microflora on Mammalian Blood Metabolites. Proc. Natl. Acad. Sci. U.S.A. 106 (10), 36983703. doi: 10.1073/pnas.0812874106

Williams, K. L., Fuller, C. R., Dieleman, L. A., DaCosta, C. M., Haldeman, K. M., Sartor, R. B., et al. (2001). Enhanced Survival and Mucosal Repair After Dextran Sodium Sulfate-Induced Colitis in Transgenic Mice That Overexpress Growth Hormone. Gastroenterology 120 (4), 925-937. doi: 10.1053/ gast.2001.22470

Wisplinghoff, H., Bischoff, T., Tallent, S. M., Seifert, H., Wenzel, R. P., and Edmond., M. B. (2004). Nosocomial Bloodstream Infections in US Hospitals: Analysis of 24,179 Cases From a Prospective Nationwide Surveillance Study. Clin. Infect. Dis. 39 (3), 309-317. doi: 10.1086/421946

Wu, S., Lim, K. C., Huang, J., Saidi, R. F., and Sears., C. L. (1998). Bacteroides Fragilis Enterotoxin Cleaves the Zonula Adherens Protein, E-Cadherin. Proc. Natl. Acad. Sci. U.S.A. 95 (25), 14979-14984. doi: 10.1073/pnas.95.25.14979

Xiong, Q. Q., Shen, T. H., Zhong, L., Zhu, C. L., Peng, X. S., He, X. P., et al. (2019). Comprehensive Metabolomic, Proteomic and Physiological Analyses of Grain Yield Reduction in Rice Under Abrupt Drought-Flood Alternation Stress. Physiol. Plant 167 (4), 564-584. doi: 10.1111/ppl.12901

Yadegari, M., Soltani, M., Saraf, Z., and Sattari., M. (2009). Evaluation of the Effect of Garlic (Iran) Extract on Th Macrophages Activity (Nitric Oxide and H2O2) Against Candida Albicans In Vitro. Mycoses 52, 126.
Yang, W., Zhou, Y., Wu, C., and Tang., J. (2016). Enterohemorrhagic Escherichia Coli Promotes the Invasion and Tissue Damage of Enterocytes Infected With Candida Albicans In Vitro. Sci. Rep. 6, 37485. doi: 10.1038/srep37485

Yan, L., Yang, C., and Tang, J. (2013). Disruption of the Intestinal Mucosal Barrier in Candida Albicans Infections. Microbiol. Res. 168 (7), 389-395. doi: 10.1016/ j.micres.2013.02.008

Yao, B., He, J., Yin, X., Shi, Y., Wan, J., and Tian., Z. (2019). The Protective Effect of Lithocholic Acid on the Intestinal Epithelial Barrier is Mediated by the Vitamin D Receptor via a SIRT1/Nrf2 and NF- אb Dependent Mechanism in Caco-2 Cells. Toxicol. Lett. 316, 109-118. doi: 10.1016/j.toxlet.2019.08.024

Yi, L., and Su, Q. (2013). Molecular Mechanisms for the Anti-Cancer Effects of Diallyl Disulfide. Food Chem. Toxicol. 57, 362-370. doi: 10.1016/ j.fct.2013.04.001

Yousuf, S., Ahmad, A., Khan, A., Manzoor, N., and Khan., L. A. (2011). Effect of Garlic-Derived Allyl Sulphides on Morphogenesis and Hydrolytic Enzyme Secretion in Candida Albicans. Med. Mycol. 49 (4), 444-448. doi: 10.3109/ 13693786.2010.539629

Zelante, T., Iannitti, R. G., Cunha, C., De Luca, A., Giovannini, G., Pieraccini, G., et al. (2013). Tryptophan Catabolites From Microbiota Engage Aryl Hydrocarbon Receptor and Balance Mucosal Reactivity via Interleukin-22. Immunity 39 (2), 372-385. doi: 10.1016/j.immuni.2013.08.003

Zhai, B., Ola, M., Rolling, T., Tosini, N. L., Joshowitz, S., Littmann, E. R., et al (2020). High-Resolution Mycobiota Analysis Reveals Dynamic Intestinal Translocation Preceding Invasive Candidiasis. Nat. Med. 26 (1), 59-64. doi: 10.1038/s41591-019-0709-7

Zhang, N., Wang, Y., Zhang, J., Liu, B., Li, G., Xin, S., et al. (2019). Diallyl Disulfide Attenuates Nonalcoholic Steatohepatitis by Suppressing Key Regulators of Lipid Metabolism, Lipid Peroxidation and Inflammation in Mice. Mol. Med. Rep. 20 (2), 1363-1372. doi: 10.3892/mmr.2019.10316

Zmora, N., Suez, J., and Elinav, E. (2019). You are What You Eat: Diet, Health and the Gut Microbiota. Nat. Rev. Gastroenterol. Hepatol. 16 (1), 35-56. doi: 10.1038/s41575-018-0061-2

Conflict of Interest: The authors declare that the research was conducted in the absence of any commercial or financial relationships that could be construed as a potential conflict of interest.

Publisher's Note: All claims expressed in this article are solely those of the authors and do not necessarily represent those of their affiliated organizations, or those of the publisher, the editors and the reviewers. Any product that may be evaluated in this article, or claim that may be made by its manufacturer, is not guaranteed or endorsed by the publisher.

Copyright (C) $2022 \mathrm{Hu}$, Huang, Zhou, Yin and Tang. This is an open-access article distributed under the terms of the Creative Commons Attribution License (CC BY). The use, distribution or reproduction in other forums is permitted, provided the original author(s) and the copyright owner(s) are credited and that the original publication in this journal is cited, in accordance with accepted academic practice. No use, distribution or reproduction is permitted which does not comply with these terms. 